\title{
First-principles study of zinc oxide honeycomb structures
}

\author{
M. Topsakal, ${ }^{1}$ S. Cahangirov, ${ }^{1}$ E. Bekaroglu, ${ }^{1}$ and S. Ciraci ${ }^{1,2}$ \\ ${ }^{1}$ UNAM-Institute of Materials Science and Nanotechnology, Bilkent University, Ankara 06800, Turkey \\ ${ }^{2}$ Department of Physics, Bilkent University, Ankara 06800, Turkey
}

(Received 12 March 2009; published 11 December 2009)

\begin{abstract}
We present a first-principles study of the atomic, electronic, and magnetic properties of two-dimensional (2D), single and bilayer $\mathrm{ZnO}$ in honeycomb structure and its armchair and zigzag nanoribbons. In order to reveal the dimensionality effects, our study includes also bulk $\mathrm{ZnO}$ in wurtzite, zincblende, and hexagonal structures. The stability of $2 \mathrm{D} \mathrm{ZnO}$, its nanoribbons and flakes are analyzed by phonon frequency, as well as by finite temperature $a b$ initio molecular-dynamics calculations. $2 \mathrm{D} \mathrm{ZnO}$ in honeycomb structure and its armchair nanoribbons are nonmagnetic semiconductors but acquire net magnetic moment upon the creation of zinc-vacancy defect. Zigzag $\mathrm{ZnO}$ nanoribbons are ferromagnetic metals with spins localized at the oxygen atoms at the edges and have high spin polarization at the Fermi level. However, they change to nonmagnetic metal upon termination of their edges with hydrogen atoms. From the phonon calculations, the fourth acoustical mode specified as twisting mode is also revealed for armchair nanoribbon. Under tensile stress the nanoribbons are deformed elastically maintaining honeycomblike structure but yield at high strains. Beyond yielding point honeycomblike structure undergo a structural change and deform plastically by forming large polygons. The variation in the electronic and magnetic properties of these nanoribbons have been examined under strain. It appears that plastically deformed nanoribbons may offer a new class of materials with diverse properties.
\end{abstract}

DOI: $10.1103 /$ PhysRevB.80.235119

PACS number(s): 73.22. $-\mathrm{f}, 75.75 .+\mathrm{a}, 63.22 .-\mathrm{m}$

\section{INTRODUCTION}

Graphene, a monolayer layer of carbon atoms in honeycomb structure, is offering exceptional properties ${ }^{1-3}$ which may lead to important applications in various fields. Normally, two-dimensional (2D) graphene is semimetallic and its electrons and holes behave like a massless Dirac fermion. Whereas 2D boron nitride (BN), ${ }^{4}$ Group III-V analog of graphene, in ionic honeycomb structure is a wide band-gap semiconductor. Unusual properties of graphene and BN nanoribbons have been revealed extensively in recent papers. $^{5-11}$ More recently, based on state-of-the-art firstprinciples calculations it was predicted that $\mathrm{Si}$ and $\mathrm{Ge},{ }^{12}$ even binary compounds of Group IV elements and III-V compounds ${ }^{13}$ can form 2D stable monolayer honeycomb structures. Earlier studies on $\mathrm{ZnO}$ and its nanowires gave first indications that graphitic $\mathrm{ZnO}$ can exists. ${ }^{14,15}$ Very thin nanosheets, ${ }^{16}$ nanobelts, ${ }^{17}$ nanotubes, ${ }^{18}$ and nanowires ${ }^{19}$ of $\mathrm{ZnO}$ have already been synthesized. Two-monolayer-thick $\mathrm{ZnO}(0001)$ films grown on $\mathrm{Ag}(111)$ were reported. ${ }^{20}$

Because of its wide band gap of $\sim 3.3 \mathrm{eV}$ and large exciton binding energy of $60 \mathrm{meV}$ leading to vast optoelectronic applications including light-emitting diodes and solar cells, ${ }^{21,22} \mathrm{ZnO}$ has been the subject of various researches. It was reported that the vacancy defects can be intentionally created by electron-irradiation method. ${ }^{23}$ It has been also reported that $\mathrm{Zn}$ vacancy induces ferromagnetism in $\mathrm{ZnO}$ thin films and nanowires without any need of doping with transition-metal atoms. ${ }^{24}$ These magnetic properties might provide superior advantages in biomedical applications because of nontoxic nature of $\mathrm{ZnO}$ as opposed to transitionmetal ions.

In this paper a comprehensive study of the atomic, electronic, and magnetic properties of monolayer, bilayer, and nanoribbons of II-VI ionic $\mathrm{ZnO}$ compound in honeycomb structures are carried out using first-principles calculations. In order to reveal the dimensionality effects, we started with the energetics and electronic energy bands of $\mathrm{ZnO}$ in different three-dimensional (3D) bulk crystalline structures and compared them with those of $2 \mathrm{D} \mathrm{ZnO}$ honeycomb structure. Our analysis based on phonon dispersions and finitetemperature $a b$ initio molecular-dynamics calculations provides evidence for the stability of free-standing 2D monolayer, bilayer, and quasi-one-dimensional (quasi-1D) nanoribbons of $\mathrm{ZnO}$ honeycomb structures. These structures can be in different local minima on the Born-Oppenheimer surface, in spite of the fact that they are not synthesized yet. We found that $2 \mathrm{D}$ monolayer and bilayer $\mathrm{ZnO}$ are nonmagnetic semiconductors but attain magnetic properties upon creation of $\mathrm{Zn}$-vacancy defect. $\mathrm{ZnO}$ nanoribbons exhibit interesting electronic and magnetic properties depending on their orientation. While armchair $\mathrm{ZnO}$ nanoribbons are nonmagnetic semiconductors with band gaps varying with their widths, bare zigzag nanoribbons are ferromagnetic metals. These electronic and magnetic properties show dramatic changes under elastic and plastic deformation. Hence, $\mathrm{ZnO}$ nanoribbons can be functionalized by plastic deformation. Results obtained in this study indicates that 2D monolayer and bilayer $\mathrm{ZnO}$ honeycomb structures and quasi-1D armchair and zigzag nanoribbons display unusual electronic, magnetic, and mechanical properties, which hold the promise of interesting technological applications.

\section{MODEL AND METHODOLOGY}

We have performed first-principles plane-wave calculations within density-functional theory (DFT) using projector augmented wave potentials. ${ }^{25}$ The exchange-correlation po- 
tential has been approximated by generalized gradient approximation (GGA) using PW91 (Ref. 26) functional both for spin-polarized and spin-unpolarized cases. Recently, spin-polarized calculations within DFT have been used successfully to investigate magnetic properties of vacancy defects in 2D honeycomb structures. Also interesting spintronic properties of nanoribbons have been revealed using spinpolarized DFT. ${ }^{6}$ The success of spin-polarized DFT calculations has been discussed in Ref. 27.

All structures have been treated within supercell geometry using the periodic boundary conditions. A plane-wave-basis set with kinetic-energy cutoff of $500 \mathrm{eV}$ has been used. The interaction between $\mathrm{ZnO}$ monolayers in adjacent supercells is examined as a function of their spacing. Since the total energy per cell has changed less than $1 \mathrm{meV}$ upon increasing the spacing from 10 to $15 \AA$, we used the spacing of $\sim 10 \AA$ in the calculations. In the self-consistent potential and totalenergy calculations the Brillouin zone (BZ) is sampled by, respectively, $(15 \times 15 \times 15),(25 \times 25 \times 1)$, and $(25 \times 1 \times 1)$ special $\mathbf{k}$ points for $3 \mathrm{D}$ bulk, 2D honeycomb, and 1D (nanoribbons) $\mathrm{ZnO}$. This sampling is scaled according to the size of superlattices. For example, BZ was sampled by $(3 \times 3$ $\times 1)$ special $\mathbf{k}$ points for defect calculations using $(7 \times 7)$ supercell of $2 \mathrm{D} \mathrm{ZnO}$ honeycomb structure. All atomic positions and lattice constants are optimized by using the conjugate gradient method, where the total energy and atomic forces are minimized. The convergence for energy is chosen as $10^{-5} \mathrm{eV}$ between two steps and the maximum HellmannFeynman forces acting on each atom is less than $0.02 \mathrm{eV} / \AA$ upon ionic relaxation. The pseudopotentials having 12 and 6 valence electrons for the $\mathrm{Zn}\left(\mathrm{Zn}: 4 s^{2} 3 d^{10}\right)$ and $\mathrm{O}$ ions $\left(\mathrm{O}: 2 s^{2} 2 p^{4}\right)$ are used. Numerical plane-wave calculations are performed by using VASP package. ${ }^{28,29}$ While all numerical calculations of structure optimization, electronic energy, and phonon dispersions are carried out within GGA using VASP, ${ }^{28,29}$ some of the calculations are checked also by using PWSCF (Ref. 30) and SIESTA (Ref. 31) softwares. Therefore all pertaining discussions are based on the results obtained by using VASP software unless it is stated otherwise.

Since DFT within GGA underestimates the band gap, frequency-dependent $G W_{0}$ calculations are carried out. ${ }^{32}$ Screened Coulomb potential, $W$, is kept fixed to initial DFT value $W_{0}$ and Green's function, $G$, is iterated five times. Various tests regarding vacuum separation, kinetic-energy cut-off potential, number of bands, $\mathbf{k}$ points and grid points are made. Final results of $G W_{0}$ corrections are obtained using $(12 \times 12 \times 1) \mathbf{k}$ points in BZ, $20 \AA$ vacuum separation, 400 $\mathrm{eV}$ cut-off potential, 160 bands, and 64 grid points. In addition to $G W_{0}$, we performed also $G W$ calculations in order to make comparison with earlier available studies. While $G W_{0}$ corrections are successfully applied to 3D and 2D $\mathrm{ZnO}$, its application to quasi-1D nanoribbons is hindered by large number of atoms.

\section{3D BULK AND 2D HONEYCOMB ZnO CRYSTAL}

We first consider 3D bulk $\mathrm{ZnO}$, which are in wurtzite (wz- $\mathrm{ZnO})$, zincblende (zb- $\mathrm{ZnO})$, and hexagonal (h- $\mathrm{ZnO}$, or graphitelike) crystals. Atoms in $\mathrm{wz}-\mathrm{ZnO}$ and $\mathrm{zb}-\mathrm{ZnO}$ struc- tures are fourfold coordinated through tetrahedrally directed $s p^{3}$ orbitals whereas the atoms in $\mathrm{h}-\mathrm{ZnO}$ crystal are threefold coordinated through $s p^{2}$ orbitals. Wurtzite structure is found to be the thermodynamically most stable phase of $\mathrm{ZnO}$. The cohesive energy per $\mathrm{Zn}-\mathrm{O}$ pair is calculated by using the expression

$$
E_{C}=E_{T}[\mathrm{ZnO}]-E_{T}[\mathrm{Zn}]-E_{T}[\mathrm{O}]
$$

in terms of the total energy of the optimized crystal structure of $\mathrm{ZnO}, E_{T}[\mathrm{ZnO}]$ per $\mathrm{Zn}-\mathrm{O}$ pair, the total energies of free $\mathrm{Zn}$ and $\mathrm{O}$ atoms $E_{T}[\mathrm{Zn}]$ and $E_{T}[\mathrm{O}]$. The equilibrium cohesive energies of $\mathrm{wz}-\mathrm{ZnO}$, $\mathrm{zb}-\mathrm{ZnO}$, and $\mathrm{h}-\mathrm{ZnO}$ structures are found to be $8.934,8.919$, and $8.802 \mathrm{eV}$ per $\mathrm{Zn}-\mathrm{O}$ pair, respectively. For wz-ZnO crystal, the hexagonal lattice constants of the optimized structure in equilibrium are $a$ $=3.280 \AA$ and $c / a=1.616$. The deviation of $c / a$ from the ideal value of 1.633 imposes a slight anisotropy in the lengths of tetrahedrally directed $\mathrm{Zn}-\mathrm{O}$ bonds. While the length of three short bonds is $2.001 \AA$, the fourth bond is slightly longer and has the length of $2.007 \AA$. The zincblende structure in $T_{d}$ symmetry has cubic lattice constant $a=3.266 \AA$ and four tetrahedrally coordinated $\mathrm{Zn}-\mathrm{O}$ bonds having uniform length, $d=2.001 \AA$. The h-ZnO structure has hexagonal lattice constants $a=3.448 \AA, c / a$ $=1.336$, and $d=1.990$. The structural parameters of these three bulk $\mathrm{ZnO}$ crystals are shown in Fig. 1. The lattice constant of wz-ZnO, $a(c / a)$ is measured between 3.247 (1.6035) $\AA$ and 3.250 (1.602) $\AA$ using different methods. ${ }^{33}$ The calculations based on $a b$ initio linear combination of atomic orbitals method with all-electron Gaussian-type basis set predict $a=3.286 \AA$ and $c / a=1.595 .{ }^{34}$ All our results related with the structural parameters are in good agreement with the experimental and theoretical values within the average error of $\sim 1 \%$. $^{33,34}$

The optimized atomic structure and corresponding electronic band structure of $3 \mathrm{D} \mathrm{ZnO}$ crystals are presented in Fig. 1. All wz-ZnO, zb-ZnO, and h-ZnO crystals are direct band-gap semiconductors with calculated band gaps being $E_{G}=0.75,0.65$, and $0.96 \mathrm{eV}$, respectively. Highest valence band has $\mathrm{O}-2 p$ character; the states of the lowest conduction band is formed from $\mathrm{Zn}-4 p$ and $\mathrm{Zn}-4 s$ orbitals. Valence band consists of two parts separated by a wide intra-band gap. The lower part at $\sim-18 \mathrm{eV}$ is projected mainly to O- $2 s$ orbitals. The upper part is due to mainly $\mathrm{Zn}-3 d$ and O-2s orbitals. The differences in the band structure of different three 3D crystals become pronounced in the lower part of the conduction band. It should be noted that the band gaps of bulk $\mathrm{ZnO}$ is highly underestimated by DFT calculations. ${ }^{35}$ The experimentally measured band gap of $\mathrm{wz}-\mathrm{ZnO}$ is $\sim 3.37 \mathrm{eV}$ at room temperature. ${ }^{33}$ We performed $G W_{0}$ calculations to correct the band gaps calculated within GGA. Our results for $\mathrm{wz}-\mathrm{ZnO}$, zb-ZnO, and $\mathrm{h}-\mathrm{ZnO}$ are, respectively, 3.29, 3.04, and $3.32 \mathrm{eV}$. As for earlier studies, calculations with $G W$ corrections reported a band gap of $3.59 \mathrm{eV}$ for $\mathrm{zb}-\mathrm{ZnO} .{ }^{36} \mathrm{All}$ electron linearized augmented planewave (LAPW) calculations predicted the band gap of $\mathrm{wz}-\mathrm{ZnO} 0.77 \mathrm{eV}$ using localdensity approximation (LDA), which is corrected by $G W$ calculations to $2.44 \mathrm{eV}^{37}$ 
(a)

\section{ZnO 3D Wurtzite}

$E_{C}=8.934 \mathrm{eV} ; E_{G}=0.75 \mathrm{eV}$
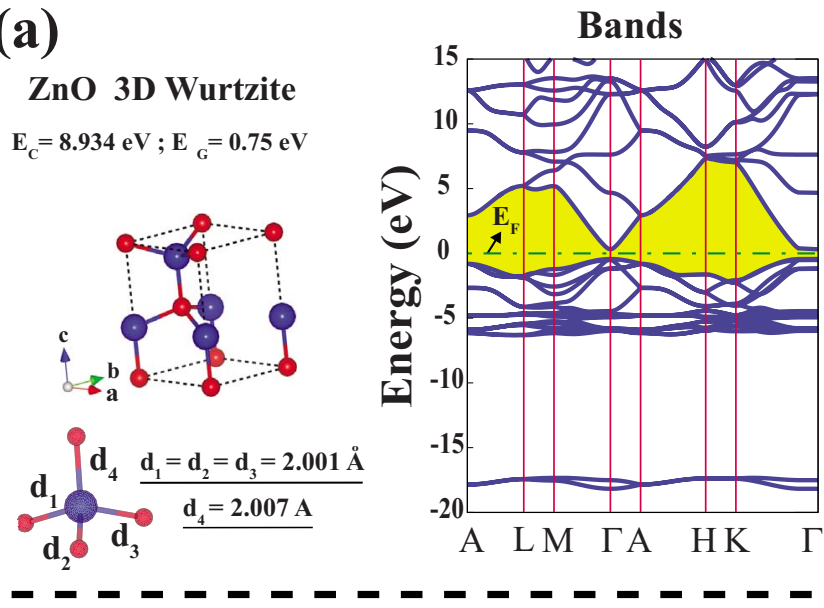

(b)

\section{ZnO 3D Zincblende}

$\mathrm{E}_{\mathrm{C}}=8.919 \mathrm{eV} ; \mathrm{E}_{\mathrm{G}}=0.65 \mathrm{eV}$

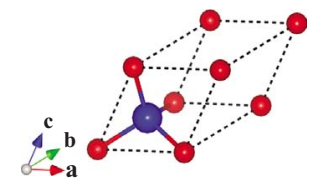

d $2_{d_{1}}^{d_{4}} \frac{d_{1}=d_{2}=d_{3}=d_{4}=d}{d=2.001 \AA}$

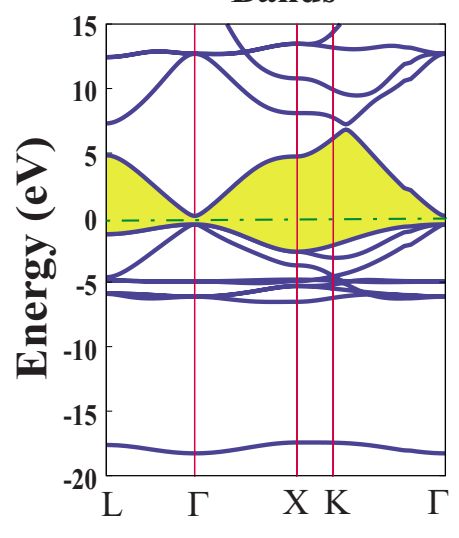

(c)

ZnO 3D Hexagonal

$\mathrm{E}_{\mathrm{C}}=8.802 \mathrm{eV} ; \mathrm{E}_{\mathrm{G}}=0.96 \mathrm{eV}$
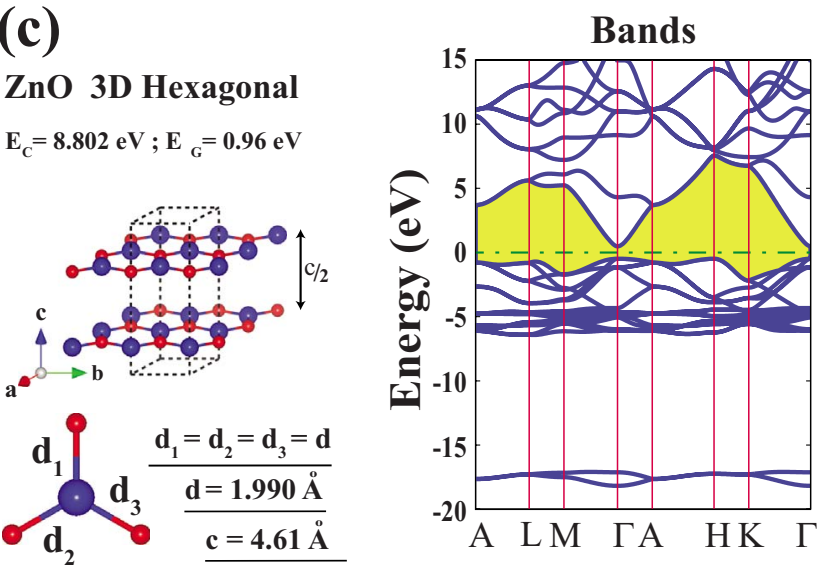

FIG. 1. (Color online) Cohesive energies $E_{C}$ per Zn-O pair, band gaps $E_{G}, \mathrm{Zn}-\mathrm{O}$ bonds, atomic and energy band structures of 3D (a) wurtzite, (b) zincblende, and (c) hexagonal crystals of bulk $\mathrm{ZnO}$. Red-small and blue-large balls correspond to $\mathrm{O}$ and $\mathrm{Zn}$ atoms, respectively. The gap between valence and conduction bands are shaded and the zero of energy is set at the Fermi level $E_{F}$. All structures including lattice constants are fully optimized.

Charge transfer from $\mathrm{Zn}$ atoms to $\mathrm{O}$ atoms is a measure of the ionicity of $\mathrm{ZnO}$ crystal. We calculated the amount of charge on constituent $\mathrm{Zn}$ and $\mathrm{O}$ atoms in 3D crystals by performing the Löwdin analysis ${ }^{38}$ in terms of the projection of plane waves into atomic orbitals. By subtracting the valencies of free $\mathrm{Zn}$ and $\mathrm{O}$ atoms from the calculated charge

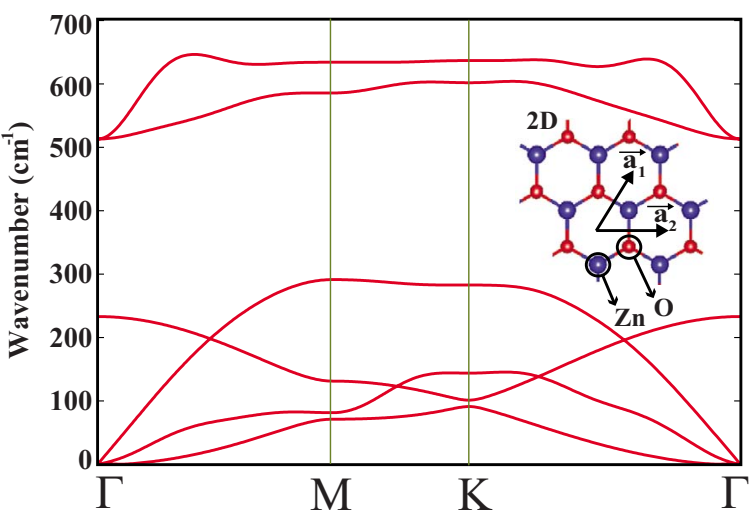

FIG. 2. (Color online) Phonon-dispersion curves calculated by force-constant method for 2D monolayer $\mathrm{ZnO}$. Atomic structure and hexagonal lattice vectors are shown by inset.

values on the same atoms in $3 \mathrm{D}$ crystals we obtain the charge transfer $\delta q$ from $\mathrm{Zn}$ to $\mathrm{O}$. The calculated value of charge transfer for $\mathrm{wz}-\mathrm{ZnO}, \mathrm{zb}-\mathrm{ZnO}$, and $\mathrm{h}-\mathrm{ZnO}$ is found to be 1.41 electrons. The values of charge transfer calculated with the Bader analysis ${ }^{39}$ are $\delta q=1.22, \delta q=1.17$, and $\delta q=1.20$ electrons for $\mathrm{wz}-\mathrm{ZnO}$, zb-ZnO, and h-ZnO, respectively. Same analysis performed with local basis set using SIESTA (Ref. 31) yields significantly lower values of charge transfer, $\delta q$ $=0.90, \delta q=0.88$, and $\delta q=0.91$ electrons for $\mathrm{wz}-\mathrm{ZnO}, \mathrm{zb}$ $\mathrm{ZnO}$, and $\mathrm{h}-\mathrm{ZnO}$, respectively. This analysis clearly indicates that a significant amount of charge is transferred from low electronegative $\mathrm{Zn}$ atom to high electronegative $\mathrm{O}$ atom. However the values of $\delta q$ may scatter owing to the ambiguities in placing boundary between $\mathrm{Zn}$ and $\mathrm{O}$ in crystalline structure.

\section{2D ZnO HONEYCOMB STRUCTURE}

The structure of monolayer $\mathrm{ZnO}$ is optimized using periodically repeating supercell having hexagonal lattice in $2 \mathrm{D}$ and the spacing of $10 \AA$ between $\mathrm{ZnO}$ planes. The optimized structure was planar and the magnitudes of the Bravais vectors of the hexagonal lattice are found to be $a_{1}=a_{2}$ $=3.283 \AA$, and the $\mathrm{Zn}-\mathrm{O}$ bond, $d=1.895 \AA$ (see Fig. 2). In contrast to $2 \mathrm{D}$ puckered honeycomb structures of $\mathrm{Si}, \mathrm{Ge}$, and compounds of III and V group elements both lying below the first row, any honeycomb structure including one element from the first row form planar honeycomb structure such as graphene, $\mathrm{BN}$, and $\mathrm{SiC} .{ }^{13} 2 \mathrm{D}$ monolayer of $\mathrm{ZnO}$ forming planar honeycomb structure is not an exception. The calculated structural parameters are significantly larger than those of graphene and $\mathrm{BN}$ honeycomb structure due to fact that $\mathrm{Zn}$ has larger radius ${ }^{40}$ than that of $\mathrm{B}, \mathrm{C}, \mathrm{N}$, and $\mathrm{O}$ atoms. The planar structure of $2 \mathrm{D} \mathrm{ZnO}$ is tested by displacing $\mathrm{Zn}$ and $\mathrm{O}$ atoms arbitrarily from their equilibrium positions by $0.5 \AA$ and subsequently by reoptimizing the structure. Upon optimization the displaced atoms have recovered their original positions in the same plane. It should be noted that the length of $\mathrm{Zn}-\mathrm{O}$ bonds of $2 \mathrm{D} \mathrm{ZnO}$ honeycomb structure is smaller than that in the $3 \mathrm{D}$ bulk (wz, zb) crystals since $s p^{2}$ bonding in the former is stronger than the tetrahedrally coordinated 
$s p^{3}$ bonding in the latter. Similar trend is also found in $\mathrm{C}$, $\mathrm{BN}$, and $\mathrm{SiC}$ honeycomb structures. The interaction between $\mathrm{ZnO}$ planes appears to slightly weaken the $\mathrm{Zn}-\mathrm{O}$ bonds of $\mathrm{h}-\mathrm{ZnO}$ crystal. As a result the length of the $\mathrm{Zn}-\mathrm{O}$ bonds becomes larger than that of $2 \mathrm{D} \mathrm{ZnO}$ honeycomb structure. The cohesive energy of 2D monolayer of $\mathrm{ZnO}$ is calculated to be $8.419 \mathrm{eV}$ per $\mathrm{Zn}-\mathrm{O}$ pair. This energy is $0.5 \mathrm{eV}$ smaller than that of $3 \mathrm{D}$ wz-ZnO.

\section{A. Atomic structure and stability analysis}

It should be noted that 2D honeycomb structure determined by the structure optimization using conjugate gradient method may not be necessarily stable. One needs more stringent tests to assure the stability of this truly 2D structure. As a matter of fact, it was argued earlier that any crystal structures consisting of a truly 2D monolayer crystals cannot be stable. ${ }^{41,42}$ Here, we summarize our analysis on the stability of planar 2D hexagonal structure of $\mathrm{ZnO}$ using calculation of phonon-dispersion curves as well as $a b$ initio finitetemperature molecular-dynamics calculations.

We calculated optical and acoustical branches of phonon frequency using the density-functional perturbation theory with plane-wave method as implemented in PWSCF software and the force-constant method ${ }^{43}$ with forces calculated using VASP. The former method includes the polarization effects so that the splitting of longitudinal- and transverse-optical modes at the $\Gamma$ point (namely, LO-TO splitting) is obtained. The force-constant method cannot yield the LO-TO splitting and is also very sensitive to the mesh size in calculating forces under atomic displacements and hence in setting up the dynamical matrix. In fact, the imaginary frequencies of ZA branch (so-called out-of-plane acoustical branch) occur around the $\Gamma$ point as an artifact of insufficient mesh size. However, all imaginary frequencies around the $\Gamma$ point (corresponding to an instability to be induced by acoustical waves with large $\lambda$ ) are removed by using very fine mesh. Earlier, imaginary frequencies of ZA modes near the $\Gamma$ point calculated for $2 \mathrm{D}$ honeycomb structures of $\mathrm{Ge}$ and III-V compounds inducing similar fortuitous instabilities for large $\lambda$ were also removed by using finer mesh. ${ }^{13}$

In Fig. 2 we present phonon-dispersion calculated for 2D monolayer of $\mathrm{ZnO}$ honeycomb structure using force-constant method, where atomic forces are calculated by VASP. Calculated phonon modes all being positive across the BZ strongly support that $2 \mathrm{D}$ monolayer of $\mathrm{ZnO}$ is a stable structure corresponding to a local minimum on the Born-Oppenheimer surface. Our phonon-dispersion curves are in agreement with those calculated in Ref. 44.

Furthermore, we have tested the stability of $2 \mathrm{D} \mathrm{ZnO}$ monolayer using finite temperature $a b$ initio moleculardynamics (MD) calculations with time steps of $2 \times 10^{-15} \mathrm{~s}$. To lift the constraints to be imposed by a small unit cell, we considered a $(7 \times 7)$ supercell of the $2 \mathrm{D}$ hexagonal $\mathrm{ZnO}$ and raised its temperature from 0 to $750 \mathrm{~K}$ in $0.1 \mathrm{ps}$. Then, we have kept the temperature of the system around $750 \mathrm{~K}$ for 2.5 ps. While all these calculations resulted in minor deformations, the honeycomb structure was not destroyed. It should be noted that these calculations may not be conclu-
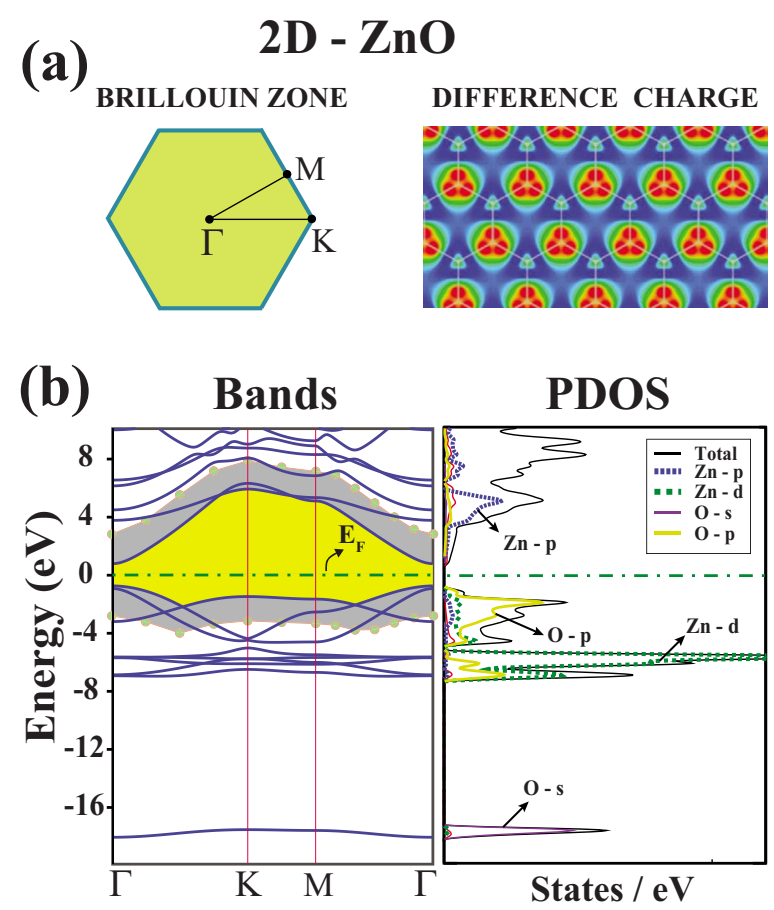

FIG. 3. (Color online) Electronic structure of the 2D monolayer of $\mathrm{ZnO}$. (a) Brillouin zone corresponding to 2D hexagonal lattice and difference charge density, $\Delta \rho$. (b) Energy band structure and density of states projected to the selected orbitals (PDOS) of constituent atoms. The band gap is shaded (painted in yellow) and the zero of energy is set at the Fermi level. Band edges corrected by $G W_{0}$ are indicated by small (light blue) circles. The band gap enlarged after $G W_{0}$ correction is shaded (painted light blue).

sive since $2.5 \mathrm{ps}$ cannot be sufficient to represent all the statistics but this picture is the best one can see from the exiguous window limited by the computational cost imposed by $a b$ initio MD method. In the following sections we will present additional arguments related with electronic structure, which further corroborate the stability of $2 \mathrm{D} \mathrm{ZnO}$ structures.

\section{B. Electronic structure}

The difference charge density and the electronic energy bands together with the partial density of states (PDOS) are presented in Fig. 3. Contour plots of total charge indicate high density around $\mathrm{O}$ atoms. The difference charge density is calculated by subtracting charge densities of free $\mathrm{Zn}$ and $\mathrm{O}$ atoms from the total charge density of $2 \mathrm{D} \mathrm{ZnO}$, i.e., $\Delta \rho$ $=\rho_{\mathrm{ZnO}}-\rho_{\mathrm{Zn}}-\rho_{\mathrm{O}}$. High-density contour plots around $\mathrm{O}$ atoms protruding toward the $\mathrm{Zn}-\mathrm{O}$ bonds indicate charge transfer from $\mathrm{Zn}$ to $\mathrm{O}$ atoms. This way the $\mathrm{Zn}-\mathrm{O}$ bond acquires an ionic character. The charge transfer from $\mathrm{Zn}$ to $\mathrm{O}, \delta q$ is analyzed by using different schemes. The charge-transfer values calculated by Löwdin method using PWSCF, Bader ${ }^{39}$ analysis using VASP, and local basis set analysis using SIESTA are, respectively, $\delta q=1.35,1.18$, and 0.87 electrons. Interestingly, as compared to 3D bulk h- $\mathrm{ZnO}$ crystal, the charge transfer from cation to anion of $2 \mathrm{D}$ monolayer $\mathrm{ZnO}$ is slightly decreased. This is due to the change from $s p^{3}$ hybrid 
orbital in $\mathrm{h}-\mathrm{ZnO}$ to $s p^{2}$ hybrid orbital in honeycomb structure.

Two dimensional $\mathrm{ZnO}$ is a direct band-gap semiconductor with a gap value of $1.68 \mathrm{eV}$. However, the actual band gap is expected to be larger. The bands are corrected using $G W_{0}$ method $^{32}$ and the direct band gap at the $\Gamma$ point of BZ is found to be $5.64 \mathrm{eV}$. Much recently, it has been reported that the band gap of $2 \mathrm{D}-\mathrm{ZnO}$ is calculated to be $3.57 \mathrm{eV}$ with $G W$ corrections. ${ }^{44}$ Similar to the bands of 3D bulk $\mathrm{ZnO}$ crystals, the upper part of the upper valance band are derived mainly from $\mathrm{O}-2 p$ orbitals whereas the lower part has $\mathrm{Zn}-3 d$ character. The bands at the edges of conduction and valence bands along the $\Gamma-K$ direction are derived from bonding and antibonding combination of $\mathrm{O}-2 p_{z}$ and $\mathrm{Zn}-4 p_{z}$ orbitals forming $\pi$ and $\pi^{*}$ states. The highest valence band along the $\Gamma-K$ direction has mainly $\mathrm{O}-2 p_{z}$ but small $\mathrm{Zn}-4 p_{z}$ orbital contribution while the lowest conduction band is composed mainly from $\mathrm{Zn}-4 p_{z}$ but small $\mathrm{O}-2 p_{z}$ orbitals. Small Zn- $4 p_{z}$ contribution is also confirmed by PDOS. Consequently, planar geometry of $2 \mathrm{D}$ monolayer of $\mathrm{ZnO}$ is expected to be attained by the bonding combination of $p_{z}$ states. The same situation occurs for 2D monolayer of $\mathrm{BN}$ honeycomb structure, which is an ionic III-V compound with wide band gap between $\pi$ and $\pi^{*}$ states. The planar stability graphene is also maintained by $\pi$ bonds.

\section{Vacancy defects and antisite}

It was shown that vacancies have remarkable effects on electronic and magnetic properties of $2 \mathrm{D}$ graphene and graphene nanoribbons. ${ }^{45-50}$ The effects of vacancies in monolayer of $\mathrm{ZnO}$ have not been treated yet. It was reported that the vacancy defects can be intentionally created by electron-irradiation method ${ }^{23}$ on $\mathrm{ZnO}$ thin films. We investigated the effects of $\mathrm{Zn}, \mathrm{O}, \mathrm{Zn}+\mathrm{O}$ divacancy and $\mathrm{O}+\mathrm{Zn}$ antisite in a periodically repeating $(5 \times 5)$ as well as $(7 \times 7)$ supercells. The vacancy-vacancy coupling in the larger $(7$ $\times 7)$ supercell was reduced significantly. Flat bands in the band gap have charge density localized at the defect site. The width of these flat bands can be taken as the measure of the strength of the direct and indirect (via the hopping through the orbitals in the lattice) vacancy-vacancy coupling. The largest width of such a band is already small (less than 50 $\mathrm{meV})$. The average energy of these flat bands from the top of the valence band can be taken as the localized states of an individual vacancy defect. Our results obtained using a (7 $\times 7)$ supercell are presented in Fig. 4 and discussed in the rest of the section.

The GGA band gap of a defect-free, 2D monolayer $\mathrm{ZnO}$ increases by $\sim 0.08 \mathrm{eV}$ in a $(7 \times 7)$ supercell including a vacancy defect. This is an artifact of the model, which mimics an individual vacancy in supercell having limited size. First we generated a $\mathrm{Zn}$ vacancy by removing a single $\mathrm{Zn}$ atom from the monolayer of $\mathrm{ZnO}$ in honeycomb structure as shown in Fig. 4(a). Upon the structure optimization, two O atoms around vacancy are departed from the plane. Similar to the vacancies in graphene and $\mathrm{BN}, \mathrm{Zn}$ vacancy induces a local magnetization in the system. Isovalue surfaces of spindensity difference $\Delta \rho$ clearly reveals the magnetism around

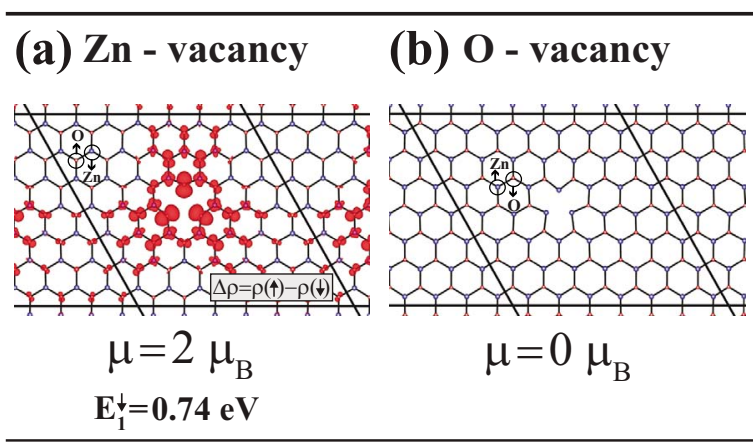

\section{(c) $\mathrm{Zn}+\mathrm{O}$ - vacancy (d) $\mathrm{Zn}+\mathrm{O}$ - antisite}

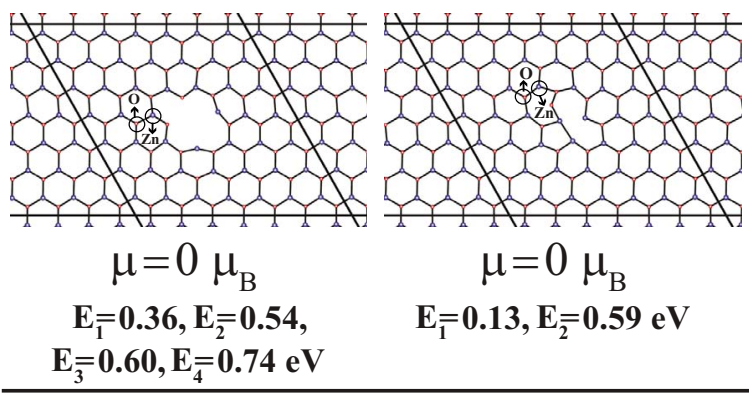

FIG. 4. (Color online) Vacancy defects in a $(7 \times 7)$ supercell of the monolayer of $\mathrm{ZnO}$. (a) Relaxed atomic structure around the $\mathrm{Zn}$ vacancy with isosurfaces of the difference charge density of spin-up and spin-down states, $\Delta \rho=\rho(\uparrow)=\rho(\downarrow)$. Energies $E_{i}$ of localized states measured from the top of the valance band and the net magnetic moment per supercell in Bohr Magneton $\mu_{B}$. Blue-large and red-small balls indicate $\mathrm{Zn}$ and $\mathrm{O}$ atoms indicated by arrows. (b) $\mathrm{O}$ vacancy. (c) $\mathrm{Zn}+\mathrm{O}$ divacancy. (d) $\mathrm{O}+\mathrm{Zn}$ antisite where neighboring $\mathrm{O}$ and $\mathrm{Zn}$ atoms are exchanged. The $(7 \times 7)$ supercells each including one type of various vacancy defects are delineated by solid lines.

vacancy. The calculated total magnetic moment is $2 \mu_{B}$ per unit cell. Note that similar $\mathrm{Zn}$ vacancy in the $3 \mathrm{D}$ bulk $\mathrm{ZnO}$ crystal induces a magnetic moment of $\sim 1 \mu_{B}$. It should be noted that the magnetic moment of $\mathrm{Zn}$ vacancy arises from the unpaired electrons of oxygen atoms around the $\mathrm{Zn}$ vacancy. Therefore it is important to determine precisely whether the vacancy-induced localized states are occupied. The positions of these localized states of $2 \mathrm{D} \mathrm{ZnO}$ calculated for the $(7 \times 7)$ supercell and their occupancy unambiguously comply with the calculated magnetic moment. The $\mathrm{Zn}$ vacancy in a repeating $(7 \times 7)$ supercell also modifies the electronic structure. The value of the band gap of defect-free $\mathrm{ZnO}$ changes from 1.68 to $1.75 \mathrm{eV}$ and a spin-up localized state appear $0.25 \mathrm{eV}$ above the top of the valence band. Figure 4(b) presents our results for $\mathrm{O}$ vacancy. Unlike the case of $\mathrm{Zn}$ vacancy, the monolayer of $\mathrm{ZnO}$ containing an $\mathrm{O}$ vacancy is nonmagnetic. $\mathrm{Zn}$ atoms around vacancy with coordination number 2 prefer to stay in the same plane of the other atoms and do not induce any magnetization. The band gap is slightly modified to $1.80 \mathrm{eV}$. As for $\mathrm{Zn}+\mathrm{O}$ divacancy in Fig. 4(c), it is again nonmagnetic. The band gap is also modified to $1.46 \mathrm{eV}$. Four occupied states associated with divacancy occur as localized states in the band gap. Finally, we consider the antisite defect. The resulting relaxed struc- 
ture is given in Fig. 4(d). The antisite $\mathrm{O}$ is pushed away from $\mathrm{Zn}$ atoms and makes bonds with a nearby $\mathrm{O}$ atom. The system does not show any magnetization. The energy of final structure with antisite defect is $\simeq 5 \mathrm{eV}$ higher (i.e., energetically less favorable) that of the defect-free 2D monolayer of $\mathrm{ZnO}$. The antisite induces two localized states in the band indicated in the figure. Also it is noted that the magnetic moments calculated for single $\mathrm{Zn}$ and $\mathrm{O}$ vacancy do not agree with Lieb's theorem, ${ }^{51}$ which predicts the amount of magnetic moments for carbon vacancies in 2D graphene. According to Lieb's theorem, the net magnetic moment per cell is expected to be $\mu=1 \mu_{B}$ for $\mathrm{Zn}$ and $\mathrm{O}$ vacancies in Fig. 4. This might be related with the ionic bonding which is different from graphene or the existence of $d$ orbitals in $\mathrm{Zn}$. On the other hand, zero magnetization for $\mathrm{Zn}+\mathrm{O}$ divacancy in Fig. $4(c)$ is in agreement with the theorem.

\section{2D ZnO bilayer}

Recently, Tusche et al. ${ }^{20}$ revealed two monolayer of $\mathrm{ZnO}$ grown on $\mathrm{Ag}(111)$ substrate using surface x-ray diffraction and scanning-tunneling microscopy. They also showed that the transition to the bulk wz-ZnO structure occurs in the 3-4 monolayer coverage. The $\mathrm{Zn}-\mathrm{O}$ bond length of the planar hexagonal structure measured $1.93 \AA$ is slightly larger than the value of $1.895 \AA$ calculated for the bond length of the monolayer of $\mathrm{ZnO}$. This situation implies that the effect of the $\operatorname{Ag}(111)$ substrate may be negligible. ${ }^{20}$

We investigated the atomic structure and stability of bilayer $\mathrm{ZnO}$ honeycomb structure. To determine the minimumenergy configuration we used periodic supercell geometry and considered five different stacking types which are $T_{1}, T_{2}$, $H_{1}, H_{2}$, and $H_{3}$. In $T_{1}$ configuration, $\mathrm{Zn}(\mathrm{O})$ atoms of the second layer are on top of the $\mathrm{Zn}(\mathrm{O})$ atoms of the first layer. $T_{2}$ is similar to $T_{1}$ except that $\mathrm{Zn}$ atoms are above $\mathrm{O}$ atoms. $H_{1}, H_{2}$, and $H_{3}$ configurations are obtained by shifting one of the layers of $T_{1}$ and $T_{2}$ on the other layer so that $\mathrm{Zn}$ or $\mathrm{O}$ atoms of the second layer are placed above the center of the hexagons in the first layer. It is $H_{1}\left(H_{2}\right)$ if $\mathrm{O}(\mathrm{Zn})$ atoms of both layers face the centers of hexagons. $H_{3}$ corresponds to the configuration where $\mathrm{O}(\mathrm{Zn})$ atom of the first(second)layer face the center of second (first) layer.

Among these five configurations we found that $T_{2}$ is energetically most favorable. $H_{3}, H_{2}, H_{1}$, and $T_{1}$ configurations have respectively $0.213,0.312,0.320$, and $0.321 \mathrm{eV}$ (per primitive cell) higher energies than $T_{2}$ configuration. The length of $\mathrm{Zn}-\mathrm{O}$ bonds as well as the charge transfer from $\mathrm{Zn}$ to $\mathrm{O}$ in $T_{2}$ configuration is slightly larger than the value of $1.895 \AA$ calculated for the bond length of the monolayer of $\mathrm{ZnO}$. This trend complies with the above discussion that the bond length increases by going from $2 \mathrm{D}$ monolayer to $3 \mathrm{D}$ bulk and implies that the effect of the $\mathrm{Ag}(111)$ substrate may be negligible. ${ }^{20}$

The bilayer binding energy among two $\mathrm{ZnO}$ layers is calculated to be $0.162 \mathrm{eV}$ (per $\mathrm{Zn}-\mathrm{O}$ pair) by subtracting the total energies of two individual monolayers from the total energy of bilayer $\mathrm{ZnO}$ in $T_{2}$ configuration. Furthermore the layer-layer separations are calculated as 4.02, 2.36, 3.80, 3.78, and $2.68 \AA$ for $T_{1}, T_{2}, H_{1}, H_{2}$, and $H_{3}$, respectively.
Hence, bilayer formation is exothermic. The calculations with LDA, which accommodate van der Waals interaction between layers better, yields $0.297 \mathrm{eV}$ (per $\mathrm{Zn}-\mathrm{O}$ pair) binding energy between two $\mathrm{ZnO}$ layers and $2.267 \AA$ layer-layer separation in $T_{2}$ structure. The layer-layer separation for $T_{2}$ is experimentally reported as $2.314 \AA{ }^{20}$ The difference between the lattice constants of the monolayer $2 \mathrm{D} \mathrm{ZnO}$ honeycomb structure and the $\mathrm{ZnO}$ bilayer is small. Owing to the relatively weak coupling between two $\mathrm{ZnO}$ monolayers, the calculated electronic structure is similar to that of singlelayer $\mathrm{ZnO}$ honeycomb structure, except that the band gap decreases to $1.44 \mathrm{eV}$ for $T_{2}$. This band gap increases to 5.10 $\mathrm{eV}$ after the $G W_{0}$ correction, which is still smaller than the $G W_{0}$ corrected band gap of 2D monolayer $\mathrm{ZnO}$.

We next address the question whether the structure of $2 \mathrm{D}$ bilayer formed by the $T_{2}$ stacking of the $\mathrm{ZnO}$ bilayer is stable is examined by the finite temperature $a b$ initio moleculardynamics and phonon-frequency calculations. $A b$ initio molecular-dynamics calculations at $750 \mathrm{~K}$ are performed with the same parameters as done for the monolayer $\mathrm{ZnO}$ in previous section indicate that the bilayer remains stable at $750 \mathrm{~K}$ after $3.0 \mathrm{ps}$.

\section{DIMENSIONALITY EFFECTS}

A comparison of $\mathrm{Zn}-\mathrm{O}$ bond length, cohesive energy, GGA bad gap, $G W_{0}$ corrected band gap calculated for 3D $\mathrm{wz}-\mathrm{ZnO}, \mathrm{zb}-\mathrm{ZnO}$, and $\mathrm{h}-\mathrm{ZnO}$, and $2 \mathrm{D}$ bilayer and monolayer of $\mathrm{ZnO}$ are presented in Table I, where interesting dimensionality effects are deduced. These dimensionality effects are believed to be crucial for better understanding of 2D crystals. Quasi-1D nanoribbons are not included to this discussion because of edge effects of the ribbon.

Three dimensional crystals have larger number of nearest neighbors and also posses larger Madelung energy as compared to $2 \mathrm{D}$ crystals. wz-ZnO appears to correspond to the global minimum of $\mathrm{ZnO}$ II-VI compound. However the energy difference between $\mathrm{wz}-\mathrm{ZnO}$ and $2 \mathrm{D}$ monolayer of $\mathrm{ZnO}$ is only $\sim 0.5 \mathrm{eV}$ and is smaller than one expects. It appears that $s p^{2}$-like bonding which is stronger than $s p^{3}$-like bonding and the $\pi$ bonding between adjacent $p_{z}$ orbitals, which contributes to stability by maintaining the planar geometry gives rise to the relatively smaller energy difference between $3 \mathrm{D}$ bulk and 2D monolayers of $\mathrm{ZnO}$. In the absence of significant $\pi$ bonding one expects that $2 \mathrm{D}$ planar $\mathrm{ZnO}$ would be buckled (puckered) for stability as found in 2D Si and GaAs honeycomb structure. ${ }^{12,13}$ Through puckered planar $s p^{2}$ orbital is dehybridized and is transformed to $s p^{3}$-like hybrid orbitals. Here we note that population of oxygen $s p^{2}$ and $p_{z}$ orbitals is larger than that of corresponding $\mathrm{Zn}$ orbitals since the former significantly higher electronegativity.

Interestingly, while GGA bad gaps of 3D $\mathrm{ZnO}$ occurs $0.75-0.96 \mathrm{eV}$, the band gaps of in $2 \mathrm{D}$ is $1.68-1.44 \mathrm{eV}$. The band gap in 2D is larger than 3D since the energy difference between $s p^{3}$-like orbitals of $\mathrm{Zn}$ and $\mathrm{O}$ ions is smaller than the energy difference in $s p^{2}$ orbitals. The latter gives rise to larger energy difference between bonding and antibonding orbitals. These band gaps are, however, underestimated by GGA since they increase approximately three times upon 
TABLE I. A comparison of bonding (or type of hybrid orbitals), the length of $\mathrm{Zn}-\mathrm{O}$ bond $d_{\mathrm{Zn}-\mathrm{O}}$, cohesive energy per Zn-O pair $E_{C}$, band gap $E_{G}$ calculated with GGA and corrected by $G_{0} W_{0}$ and $G W_{0}$ in the present study, earlier $G W$ calculations, and experimental value.

\begin{tabular}{lcccccccc}
\hline \hline ZnO & Bonding & \multicolumn{1}{c}{$d_{\text {Zn-O }}$} & $\begin{array}{c}E_{C} \\
(\mathrm{eV} / \text { atom) }\end{array}$ & GGA & $G_{0} W_{0}$ & $G W_{0}$ & $G W$ & Experiment \\
\hline 3D $w z$ & $s p^{3}$ & $2.001-2.007$ & 8.934 & 0.75 & 2.76 & 3.29 & $2.44^{\mathrm{a}}$ & $3.37^{\mathrm{d}}$ \\
3D $z b$ & $s p^{3}$ & 2.001 & 8.919 & 0.65 & 2.53 & 3.09 & $3.59^{\mathrm{b}}$ & \\
3D $h$ & $s p^{2}+p_{z}+V d W$ & 1.999 & 8.802 & 0.96 & 2.84 & 3.32 & & \\
2D bilayer & $s p^{2}+p_{z}+V d W$ & 1.932 & 8.640 & 1.44 & 4.45 & 5.10 & & \\
2D monolayer & $s p^{2}+p_{z}$ & 1.895 & 8.478 & 1.68 & 4.87 & 5.64 & $3.57^{\mathrm{c}}$ & \\
\hline \hline
\end{tabular}

Reference 37.

${ }^{\mathrm{b}}$ Reference 36.

${ }^{\mathrm{c}}$ Reference 44.

${ }^{\mathrm{d}}$ Reference 33 .

$G W_{0}$ corrections. It appears that band-gap values corrected by $G W_{0}$ is closer to experimental value and hence superior to $G W$ correction. As for as $d_{\mathrm{Zn-O}}, E_{C}$ and $E_{G}$ are concerned, 3D $\mathrm{h}-\mathrm{ZnO}$ is intermediate between $3 \mathrm{D}$ and $2 \mathrm{D}$ structures. Also calculated values of bilayer $\mathrm{ZnO}$ are slightly closer to $3 \mathrm{D}$ than those of 2D monolayer of $\mathrm{ZnO}$.

Charge transfer from $\mathrm{Zn}$ to $\mathrm{O}$ is crucial for dimensionality effects but difficult to calculate precisely. Charge transfer, $\delta q$, calculated using three different schemes, namely, Löwdin, Bader, and Siesta, yield different but consistent values. For example, the Löwdin values occur around 1.4 electrons for 3D but relatively smaller value of 1.35 electrons for 2D. Bader analysis yields $1.22-1.20$ electrons for 3D and relatively smaller value of 1.18 electrons for 2D. As for Siesta, $3 \mathrm{D}$ values, they are 0.90 electrons for $3 \mathrm{D}$ but 0.87 electrons for 2D. Excluding the paradoxical situation with chargetransfer values of $\mathrm{zb}-\mathrm{ZnO}$ occurring close to those of $2 \mathrm{D}$ $\mathrm{ZnO}$, the charge transfer between $\mathrm{Zn}$ and $\mathrm{O}$ is slightly smaller in $2 \mathrm{D}$ than $3 \mathrm{D}$.

\section{ZnO NANORIBBONS}

In this section, we consider bare and hydrogen-passivated armchair (a- $\mathrm{ZnO})$ and zigzag (z-ZnO) $\mathrm{ZnO}$ nanoribbons. These nanoribbons are specified according to their width given in terms of $n$ number of $\mathrm{Zn}-\mathrm{O}$ pairs in their unit cells. Hence, $\mathrm{z}-\mathrm{ZnO}(n)$ indicates a zigzag $\mathrm{ZnO}$ nanoribbons having $n \mathrm{Zn}-\mathrm{O}$ pairs in their unit cell. We investigate their electronic, magnetic, and mechanical properties. First, we start with the stability analysis of these nanoribbons.

\section{A. Stability analysis}

We have analyzed the stability of the bare armchair $\mathrm{ZnO}$ nanoribbons having $n=9 \mathrm{Zn}-\mathrm{O}$ atom pairs in the unit cell using the force-constant method and the finite-temperature molecular-dynamics calculations. The dynamical matrix was generated using the forces calculated in seven unit cells of a- $\mathrm{ZnO}(9)$. Results of this calculation were presented in Fig. 5(a). Phonon-dispersion curves of quasi-1D nanoribbons, in general, show profiles expected from the folding of $2 \mathrm{D}$ phonon-dispersion curves. Modes appearing above
$700 \mathrm{~cm}^{-1}$, however, are not expected from this folding. They were attributed to edge effects and reconstructions.

Right panel of Fig. 5(a) presents the acoustic region in ten times magnified scale. There are four acoustic modes, dispersion curves of which go to zero as $\mathbf{k} \rightarrow 0$. To get exactly zero value, we have imposed the symmetries originating from translational and rotational invariance on the dynamical matrix. To impose these symmetries we have tuned the forceconstant matrix elements so that all forces on all atoms are zero when the whole structure is shifted in three dimensions or slightly rotated along the axis passing through the middle of the nanoribbon.

Both the longitudinal and transverse-acoustic modes have linear dispersions near the $\Gamma$ point. Group velocity of LA mode is slightly higher than that of TA mode. Out of plane ZA and twisting acoustical TW modes ${ }^{52}$ have quadratic dispersion near the $\Gamma$ point, which is attributed to the rapid decay of the force constants with increasing neighbor distance. In fact there are some imaginary frequencies in ZA mode near the $\Gamma$ point. The absolute value of these frequencies do not exceed $0.5 \mathrm{~cm}^{-1}$ and they are purely an artifact of the precision of the numerical calculation. Using a finer mesh makes these imaginary frequencies disappear.

We also have calculated vibrational modes of the finite patch of $\mathrm{ZnO}$ having the length of five unit cells and the width of $n=9$. Zigzag edges of this flake were saturated by hydrogen atoms to eliminate the magnetism and to simplify the numerical computations. Vibrational spectrum of this structure had no imaginary frequencies implying the fact that finite-size flakes of $2 \mathrm{D} \mathrm{ZnO}$ honeycomb structure is stable. The DOS of calculated phonon frequencies are presented in Fig. 5(b). Note that, DOS calculated for three different honeycomb systems are similar. Owing to the edge effects the gap between the acoustical and optical branches of the ribbon is reduced. Similar effect as well as broadening of discrete-mode frequencies cause DOS of the flake to deviate significantly from that of $2 \mathrm{D}$ honeycomb structure in the gap. The vibrational modes attributed to strong $\mathrm{Zn}-\mathrm{H}$ and $\mathrm{O}-\mathrm{H}$ bonds are centered, respectively, at 1830 and $3700 \mathrm{~cm}^{-1}$. These modes are not shown since these frequencies are beyond the range of Fig. 5(b).

$A b$ initio molecular dynamic calculations were also car- 


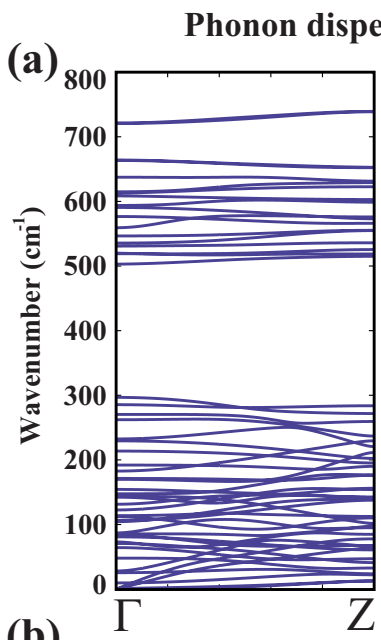

(b)

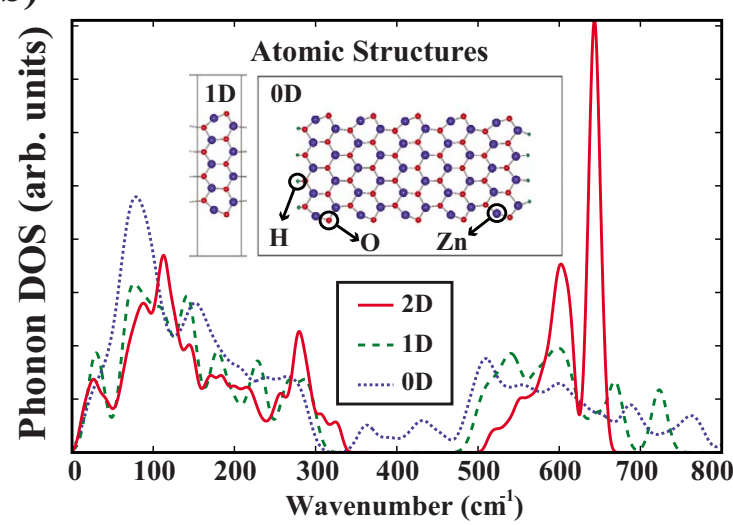

FIG. 5. (Color online) (a) Phonon-dispersion curves of a- $\mathrm{ZnO}(9)$ calculated by force-constant method. Right panel presents the magnified view of the low-lying modes. Here the well-known out-ofplane, transverse, and longitudinal acoustical modes are labeled, respectively, as ZA, TA, and LA. The fourth acoustical twisting mode, which is indigenous to quasi-1D nanoribbons, is labeled as TW, because this mode corresponds to the twisting of the nanoribbon along the axis passing through the middle of the ribbon in the infinite direction. (b)DOS calculated for 2D planar $\mathrm{ZnO}$ (shown by solid lines); armchair a- $\mathrm{ZnO}(9)$ (shown by dashed lines); and a finite-size flake, i.e., a 5 unit cell long a- $\mathrm{ZnO}(9)$ (shown by dotted lines). The discrete frequencies due to finite-size structure are Gaussian broaden with $\sigma=8 \mathrm{~cm}^{-1}$ in order to compare with continuous DOS of 2D layer and quasi-1D ribbon. Quasi-1D (nanoribbon) and $\mathrm{OD}$ (flake) honeycomb structures are shown by inset.

ried out for a- $\mathrm{ZnO}(9)$ and a finite-size patch of it. To eliminate constraints to be imposed by small unit cell, the infinite nanoribbon is treated by a supercell composed of five unit cells. Both nanoribbons are kept at $750 \mathrm{~K}$ for $3.5 \mathrm{ps}$. As a result, infinite, periodic structure of a- $\mathrm{ZnO}(9)$ composed of five unit cells had minor reconstructions at the edges while its honeycomb structure was preserved. Finite structure had the similar pattern at the armchair edges but the reconstructions at the hydrogen-saturated zigzag edges were more pronounced. These reconstructions made the whole system bend but again the honeycomb structure was preserved around the central region of the nanoribbon. The results of this analysis are interpreted that finite-size $\mathrm{ZnO}$ nanoribbons are stable. (a)

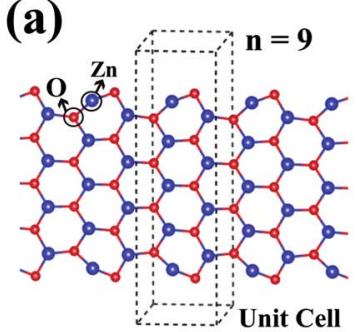

(b)
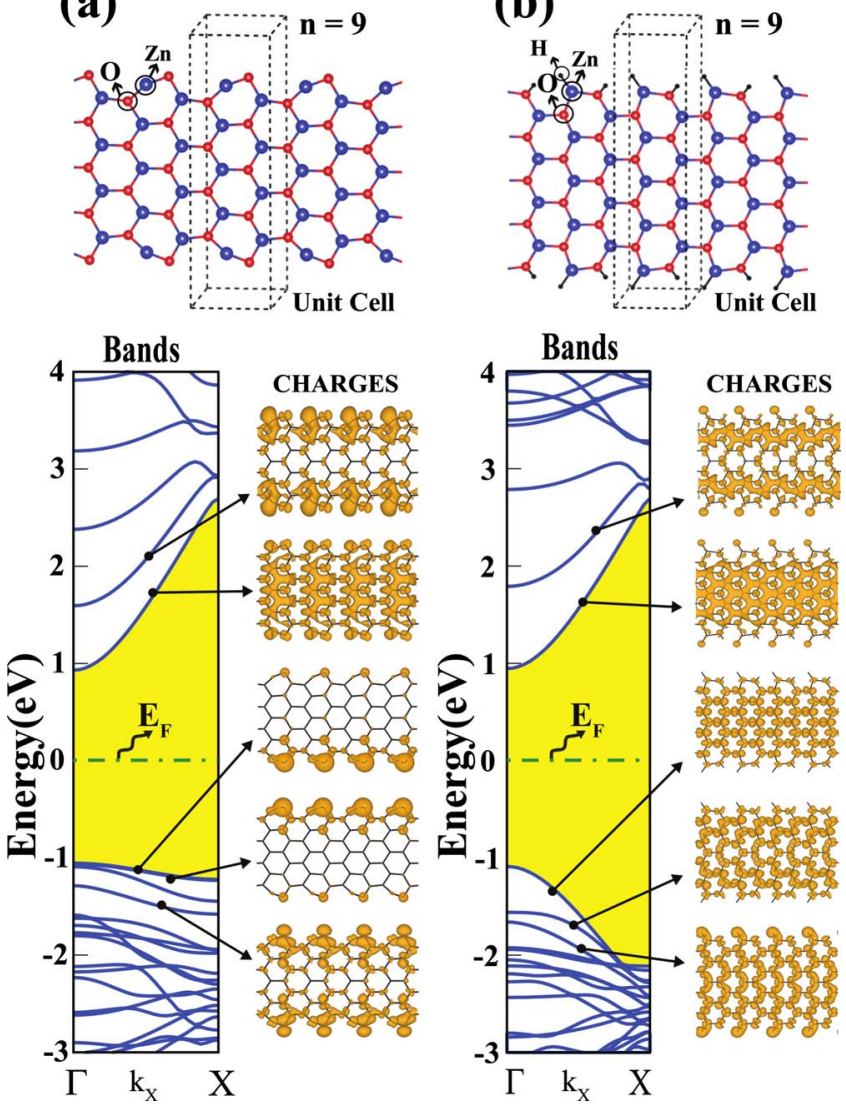

FIG. 6. (Color online) Atomic and electronic structure of bare and hydrogen-terminated armchair nanoribbons a- $\mathrm{ZnO}(9)$. (a) Atomic structure with unit cell comprising $n=9 \mathrm{Zn}-\mathrm{O}$ atom pairs, energy band structure and charge-density isosurfaces of selected states of bare nanoribbons. The charge of the bands of edge states are localized at the edge atoms. (b) Same as (a) but for hydrogenpassivated nanoribbon. Large, medium, and small balls indicate $\mathrm{Zn}$, $\mathrm{O}$, and $\mathrm{H}$ atoms, respectively. Unit cells are delineated by dashed lines.

\section{B. Electronic and magnetic properties}

Bare and hydrogen-terminated $\mathrm{a}-\mathrm{ZnO}$ nanoribbons are nonmagnetic semiconductors with direct band gaps which are relatively larger than that of the monolayer $\mathrm{ZnO}$. Figures 6(a) and 6(b) show the atomic and electronic structure of bare and hydrogen-terminated $\mathrm{a}-\mathrm{ZnO}(n)$ with $n=9$. The atoms at the edges of the bare $\mathrm{a}-\mathrm{ZnO}$ are reconstructed; while one edge atom, $\mathrm{Zn}$ is lowering, adjacent edge atom, $\mathrm{O}$ is raised. The energy band gap calculated with GGA is direct and $1.92 \mathrm{eV}$ wide and is larger than the band gap of $2 \mathrm{D}$ monolayer $\mathrm{ZnO}$. Band-decomposed charge-density analysis of a- $\mathrm{ZnO}(9)$ is presented in Fig. 6. The highest valance bands are degenerate and their charge accumulates on oxygen edge atoms for bare ribbon. The third band from the top of valence band is also associated with edge states. On the other hand, the charge of the lowest conduction band is distributed uniformly in the ribbon. The charge of the second conduction band is mostly distributed at the dangling bonds on the edges of the ribbon.

The passivation of $\mathrm{Zn}$ and $\mathrm{O}$ atoms at the edges by hydrogen atoms gives rise to changes in the electronic band 


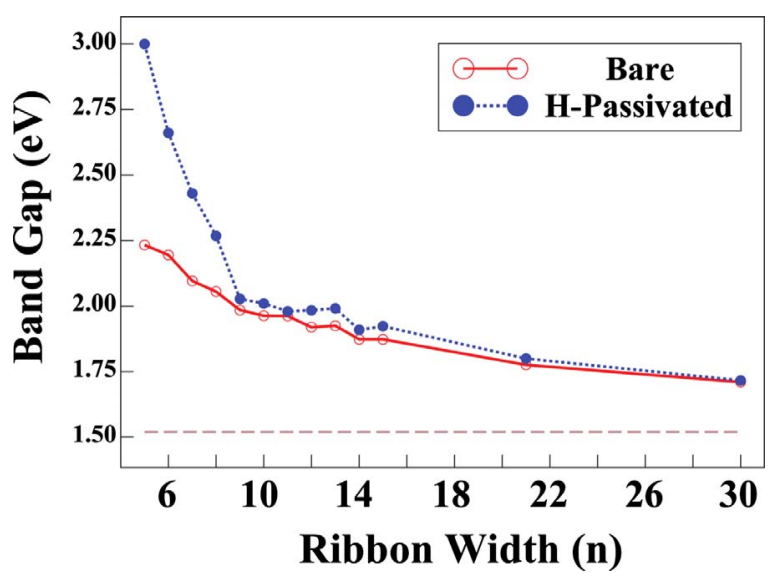

FIG. 7. (Color online) Variation in the calculated band gaps of bare and hydrogen-passivated $\mathrm{ZnO}$ armchair nanoribbons with their widths $n$. Dashed line indicates the band gap of the infinite 2D ZnO.

structure. The bond lengths $\mathrm{Zn}-\mathrm{H}$ and $\mathrm{O}-\mathrm{H}$ bonds are calculated to be 1.53 and $0.97 \AA$. As seen in Fig. 6(b), upon passivation with hydrogen atoms, the reconstruction of edge atoms are weakened. At the end the edge state bands are discarded from the band gap and replaced by dispersive bands having charge distributed uniformly in the ribbon. The band gap of $\mathrm{H}$-passivated a- $\mathrm{ZnO}(9)$ slightly increases to $\sim 1.98 \mathrm{eV}$.

The variation in the band gap $E_{G}$ for the bare and hydrogen saturated armchair nanoribbons as a function of $n$ is given in Fig. 7. The band gaps are large for small $n$ but approaches to those of 2D honeycomb structure as the width $n \rightarrow \infty$. This is an indication of the quantum-size effect. For $n<9$, the value of the band gap of hydrogen-passivated $\mathrm{a}-\mathrm{ZnO}$ is significantly larger than that of bare ribbons; the difference practically disappears for $n>20$. In contrast to graphene and $\mathrm{BN}$ nanoribbons, family-dependent variations in band gap ${ }^{53}$ is absent in Fig. 7. The variation in the band gap with $n$ is an important property, which may lead to formation of quantum dot or multiple quantum wells through the size modulation. ${ }^{8}$

Earlier it has been reported ${ }^{54,55}$ that all a-ZnO nanoribbons are semiconductors with a constant band gap of $2 \mathrm{eV}$. In our case, however, the band gaps of nanoribbons, especially for $n<10$, display apparent dependency on $n$. The tendency of the decrease in the band gaps to the $2 \mathrm{D} \mathrm{ZnO}$ band gap is also observed when $n$ is increased.

In contrast to a- $\mathrm{ZnO}$ nanoribbons, all zigzag nanoribbons $(\mathrm{z}-\mathrm{ZnO})$ we investigated in this work (namely, $n=5,7,9$ ) show metallic character regardless of their widths. On the other hand, while all a-ZnO nanoribbons are nonmagnetic, bare $\mathrm{z}-\mathrm{ZnO}$ nanoribbons are magnetic due to edge states. The magnetic properties of $\mathrm{z}-\mathrm{ZnO}$ depend on whether the edge atoms are passivated with hydrogen. Our results regarding with the bare and hydrogen-passivated $\mathrm{z}-\mathrm{ZnO}(n)$ nanoribbons for $n=9$ are presented in Figs. 8(a)-8(c). In Fig. 8(a), while the upper edge of the ribbon is made by $\mathrm{O}$ atoms, the lower edge terminates with $\mathrm{Zn}$ atoms. The bare $\mathrm{z}-\mathrm{ZnO}$ nanoribbon is magnetic since the spin-polarized calculations yield the total energy, which is energetically $34 \mathrm{meV}$ favorable than that obtained from spin-unpolarized calculations.
The total magnetic moment of the system was calculated as $0.57 \mu_{B}$ per unit cell. Figure 8(b) shows total DOS and band structure of bare $\mathrm{z}-\mathrm{ZnO}(9)$ together with isosurface charge densities of selected bands. Bare $\mathrm{z}-\mathrm{ZnO}(9)$ have isosurfaces of difference charge density $\Delta \rho$ occur around $\mathrm{O}$ edge atoms due to unpaired $\mathrm{O}-2 p$ orbitals. Clearly, bare $\mathrm{z}-\mathrm{ZnO}$ is a ferromagnetic metal. These results are in agreement with those predicting that the ferromagnetic behavior of $\mathrm{ZnO}$ nanoribbons due to unpaired spins at the edges is dominated by oxygen atoms. ${ }^{54,55}$

The band structure of the nanoribbon gives us further information about the magnetism of the system. When we plot the spin-up and spin-down bands we observe the splitting of these bands around Fermi level. The conduction and lower valance bands are degenerate for spin-up and spin-down states, some of which are shown with yellow isosurface charge densities in the figure. We also plotted charge density of two states of spin-split nondegenerate bands. Spin-down state is half filled while spin-up state is totally filled. Furthermore, their charges are confined to the $\mathrm{O}$ edge of the ribbon. The spin polarization at the Fermi level is calculated in terms of density of spin-up and spin-down states, $D\left(\uparrow, \downarrow, E_{F}\right)$, namely, $\quad P\left(E_{F}\right)=\left|D\left(\uparrow, E_{F}\right)-D\left(\downarrow, E_{F}\right)\right| /\left[D\left(\uparrow, E_{F}\right)+D\left(\downarrow, E_{F}\right)\right]$ and is around $80 \%$ for spin-down states although the spin-up states are majority spins. This suggests $\mathrm{z}-\mathrm{ZnO}(n)$ with high spin polarization at $E_{F}$ can operate as spin-valve device.

As we discussed in Sec. IV C, the magnetism of $\mathrm{Zn}$ vacancy in the monolayer $\mathrm{ZnO}$ is directly related with $\mathrm{O}$ atoms having unpaired $2 p$ orbitals. Similarly, bare $\mathrm{z}-\mathrm{ZnO}$ nanoribbons have also magnetic states on $\mathrm{O}$ terminated edge of the ribbon. The splitting of spin-up and spin-down bands around Fermi level is removed when the edges of bare $\mathrm{z}-\mathrm{ZnO}(9)$ in Fig. 8(a) is passivated by hydrogen. The resulting structure is again metallic but nonmagnetic. The electronic properties of the $\mathrm{z}-\mathrm{ZnO}(9)$ ribbon passivated with hydrogen is presented in Fig. 8(c). Two bands just below the Fermi level are localized on the $\mathrm{Zn}$ edge of the ribbon and lowest conduction band is localized on the $\mathrm{O}$ edge of the ribbon. Similar results are also obtained for $n=5$ and $n=7$. The situation regarding the magnetism of bare $\mathrm{z}-\mathrm{ZnO}$ is somewhat different from that of the bare zigzag graphene nanoribbons, which have ferromagnetic coupling along the edges but antiferromagnetic coupling between the edges. ${ }^{5}$ Moreover, unlike the case in $\mathrm{z}-\mathrm{ZnO}$ nanoribbons, the magnetism of zigzag graphene nanoribbons are not destroyed upon termination of edges with hydrogen atom.

An important feature of zigzag $\mathrm{ZnO}$ nanoribbons is that charged $\mathrm{Zn}$ and $\mathrm{O}$ atoms terminate different edges and thus induce sizable electric-dipole moments. For the bare $\mathrm{z}-\mathrm{ZnO}(9)$ the dipole moment is calculated to be $0.78($ electron $\times \AA$ ) per cell. Upon $\mathrm{H}$ termination of $\mathrm{Zn}$ and $\mathrm{O}$ edge atoms the dipole moment increases to 1.07 (electron $\times \AA)$ per cell and its direction is reversed. While the dipole effects are included in the electronic-structure calculations of $\mathrm{H}$-terminated $\mathrm{z}-\mathrm{ZnO}(9)$ nanoribbons, the band gap underestimated by GGA may effect its metallicity. Unfortunately, $G W_{0}$ corrections cannot be applied due to large number of atoms. 
(a)

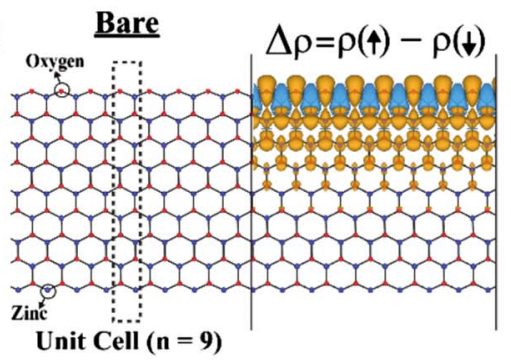

(c)

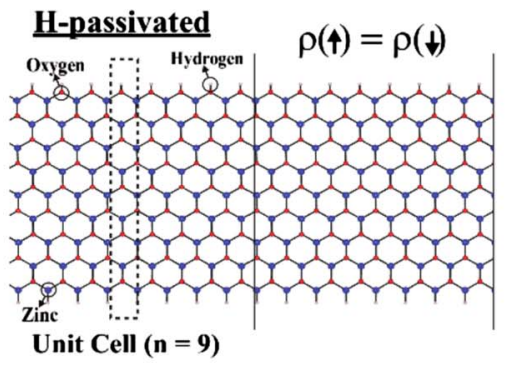

(b) Dos

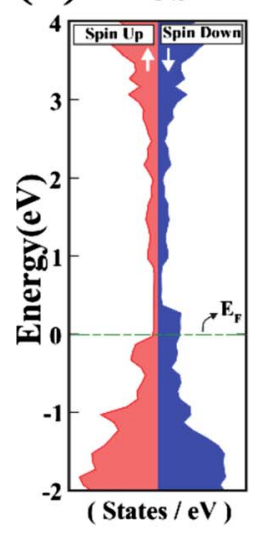

BANDS CHARGES

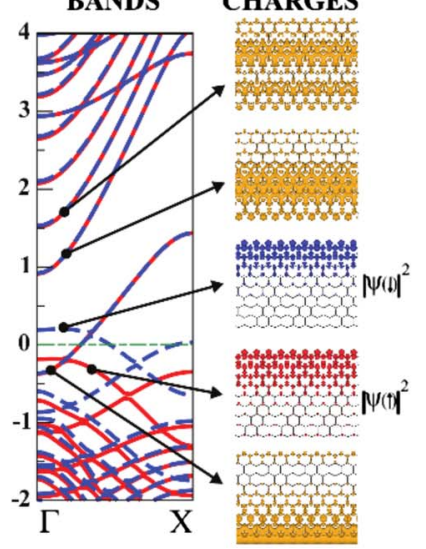

(d)

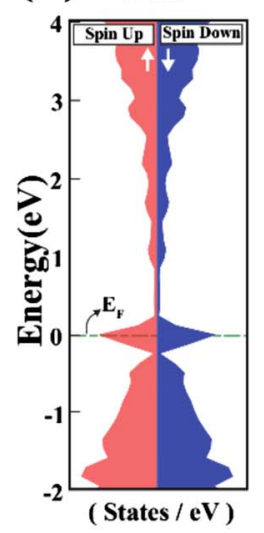

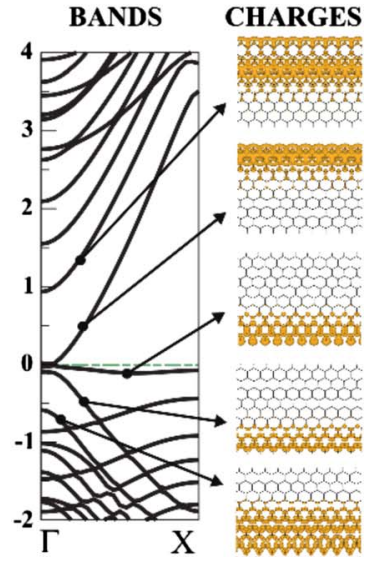

FIG. 8. (Color online) Bare zigzag nanoribbon $\mathrm{z}-\mathrm{ZnO}(n)$ with $n=9$ : (a) honeycomb structure with primitive unit cell delineated and difference charge density $\Delta \rho$ of spin-up and spin-down states. (b) Total density of spin-up and spin-down states (DOS), energy band structure, and isosurface charge density of selected spin states. Large, medium, and small filled balls indicate $\mathrm{Zn}$, O, and $\mathrm{H}$ atoms, respectively. (c) and (d) are same as (a) and (b). Yellow-light and blue-dark densities are for spin-up and spin-down states. Similarly red-continuous and blue-dashed curves are spin-up and spin-down spin bands. Black lines are for nonmagnetic (spin-paired) bands.

\section{Mechanical properties of $\mathrm{ZnO}$ nanoribbons}

The response of the nanoribbons to the applied uniaxial stress is crucial and provides information on the strength of the nanoribbons. More recently, the response of graphene to strain (or to tension) in the elastic deformation range has been an active field of study. For example, recent works ${ }^{56}$ indicated the effect of deformation on the electronic properties for band-gap engineering. Moreover theoretical studies ${ }^{57}$ have shown that carbon atomic chain can be derived from graphene under tension. Jin et al. ${ }^{58}$ showed that carbon atomic chains can be derived from graphene by electron irradiation inside a transmission electron microscope. Therefore, the response of $\mathrm{ZnO}$ nanoribbons to tension is of crucial importance.

Owing to ambiguities in defining the cross section of the ribbon one cannot determine the Young's modulus rigorously. We examined the variation in the strain energy, $E_{s}(\epsilon)$ $=E_{T}(\epsilon)-E_{T}(\epsilon=0)$ with respect to the applied uniaxial strain, $\epsilon=\Delta c / c, c$ being the lattice constant along the ribbon axis. The variation in $E_{s}(\epsilon)$ includes information regarding the mechanical properties of the ribbon. For example, force constant, $\kappa=\partial^{2} E_{T} / \partial c^{2}$ is obtained from the variation in $E_{s} . \kappa$ is an observable and can specify the strengths of ribbons unambiguously. The effects of two edges due to incomplete hexagons becomes important for narrow nanoribbons but decrease with increasing width. In-plane stiffness $C$, which is independent of the thickness, can also be used instead of
Young's modulus. Defining $A_{0}$ as the equilibrium area of the system, $C$ can be given as

$$
C=\frac{1}{A_{0}}\left(\frac{\partial^{2} E_{s}}{\partial \epsilon^{2}}\right) .
$$

By choosing a reasonable thickness " $h$, "Young's modulus can be recalled as $Y=C / h$. The thickness value around $3.34 \AA$ was used in order to evaluate the elastic properties of SWNTs and graphene by some works in literature. ${ }^{59-61} \mathrm{We}$ note that while $C$ is unique for $2 \mathrm{D}$ infinite (periodic) honeycomb structure of $\mathrm{ZnO}$, for nanoribbons it depends on how the width of the ribbon is taken in determining $A_{0}$. In fact, the width of the a- $\mathrm{ZnO}$ nanoribbon cannot be determined straightforwardly. It is not clear whether the distance is from $\mathrm{Zn}$ at one edge to $\mathrm{Zn}$ at the other edge or from $\mathrm{O}$ at one edge to other O. One can also take the average of these two or consider charge spill over from these edge atoms. Hence the value of $C$ is subject to small changes depending on how the width of the nanoribbon is taken. This uncertainty, however, diminish as the width increases; eventually the value of $C$ converges to a fixed value. In this respect, once the width is fixed $C$, can be determined from $\kappa$.

We considered three nanoribbons, a- $\mathrm{ZnO}(9)$, a- $\mathrm{ZnO}(15)$, and $\mathrm{z}-\mathrm{ZnO}(9)$. In order to eliminate the constraints imposed by the periodic boundary conditions of small unit cell, calculations are performed using a supercell composed of four 
(a)
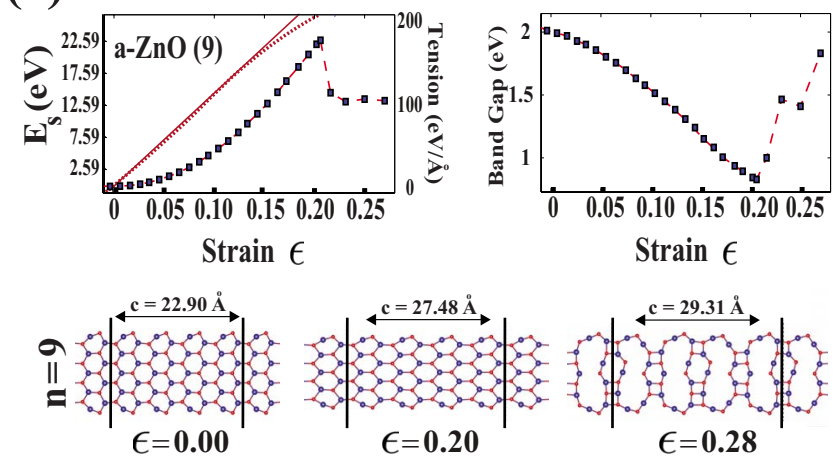

(b)
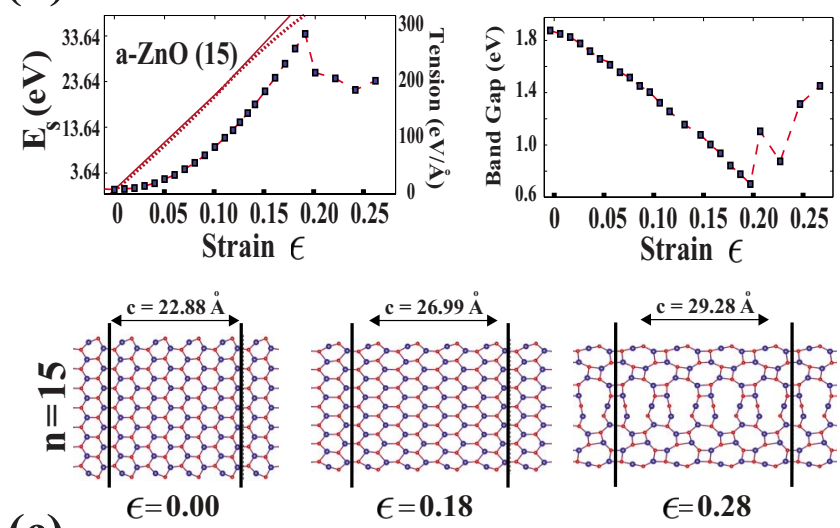

$\epsilon=0.18$

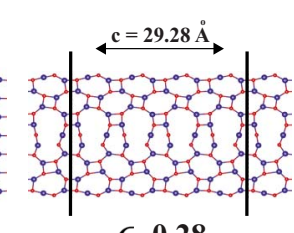

(c)

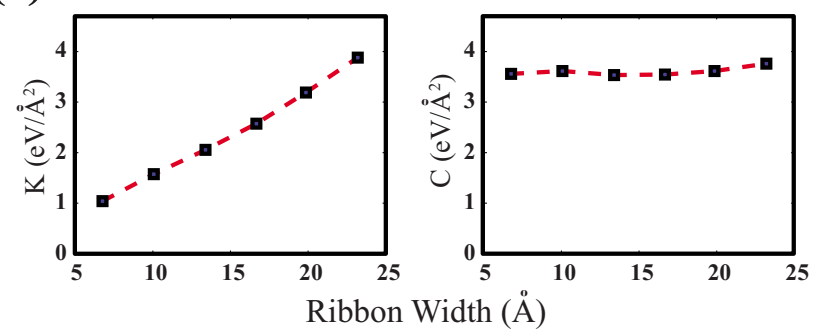

FIG. 9. (Color online) (a) Variation in the strain energy $E_{s}$ and band gap $E_{G}$ of the bare a- $\mathrm{ZnO}(n)(n=9)$ with the applied uniaxial strain, $\epsilon$ are shown by a curve with squares. Calculated tension shown by tin (red) curve is initially linear but deviates from linearity for large $\epsilon$. Atomic structure for three different values of strain. The boundaries of the supercells comprising four unit cells and corresponding lattice constants $c$ are indicated by vertical lines. (b) Same as (a) but for bare a- $\mathrm{ZnO}(n)(n=15)$. (c) Variation in force constant, $\kappa$ and in-plane stiffness, $C$ of a-ZnO with the width $w$ of the nanoribbons.

primitive unit cells for a- $\mathrm{ZnO}$ and five unit cells for $\mathrm{z}-\mathrm{ZnO}$. The size of supercells is determined based on certain tests. Figures 9(a) and 9(b) show the variation in the strain energy $E_{s}(\epsilon)$ and band gap $E_{G}(\epsilon)$ with the applied strain, $\epsilon$ for both ribbons, a- $\mathrm{ZnO}(9)$ and a- $\mathrm{ZnO}(15)$. They display a parabolic $E_{s}$ vs $\epsilon$ curves up to the strains $\epsilon \sim 0.13$. Beyond these strain values, energy vs strain variation becomes elastic but anharmonic. In the elastic range, the ribbons preserve their honeycomblike structure but the hexagons are elongated uniformly along the direction of the strain. Zones of harmonic and anharmonic variation in $E_{s}(\epsilon)$ can be better deduced by calculating the tension force, $F_{T}(\epsilon)=-\partial E_{S}(\epsilon) / \partial c . F_{T}(\epsilon)$ is linear for $0 \leq \epsilon \leq \sim 0.13$ in the elastic-harmonic range but becomes nonlinear for $\sim 0.13 \leq \epsilon$ as seen in Figs. 9(a) and 9(b). If the applied tension is released, the elastic deformation in both harmonic and anharmonic ranges disappears and the ribbon returns to its original equilibrium geometry.

The elastic deformation range of $\mathrm{a}-\mathrm{ZnO}(9)$ and $\mathrm{a}-\mathrm{ZnO}(15)$ ends with a sharp fall of the total energy. This point is identified as the yielding point of the ribbon occurring at $\epsilon$ $\simeq 0.20$. The yielding points are followed with structural transformation, where honeycomb cage structure starts to undergo a change and part of the strain energy is relieved. Once the yielding point is passed the ribbon can stretch under significantly low tension and some fraction of the deformation will be permanent and irreversible if the tension is released. Similar behavior occurs when a nanowire of metals, such as $\mathrm{Au}$ or $\mathrm{Cu}$; following an order-disorder transformation the elastic deformation ends and the wire is elongated by one lattice constant (or it deforms plastically). However, in contrast to the present case, the nanowire attains again the ordered state and start to deform elastically. ${ }^{62}$

The force constant is calculated to be $\kappa=2.05$ and $3.88 \mathrm{eV} / \AA^{2}$ for a- $\mathrm{ZnO}(9)$ and a- $\mathrm{ZnO}(15)$, respectively. The increase in $\kappa$ from 2.05 to $3.88 \mathrm{eV} / \AA^{2}$ is due to the increase in the width from $n=9(\sim 12.81 \AA)$ to $n=15(\sim 22.65 \AA)$. Note that if the Hooke's law were valid for these nanoribbons, the ratio $\kappa(15) / \kappa(9)$ would be equal to the ratio of widths, namely, $22.65 / 12.81=\sim 1.77$. The calculated value $(3.88 / 2.05=1.89)$ is slightly higher. The discrepancy arises from the edges of the ribbons, which respond to tension differently from the central region. Therefore, deviation from the Hooke's law becomes more serious as $n$ decreases but diminish as $n \rightarrow \infty$. $\kappa$ values of a- $\mathrm{ZnO}(9)$ and a- $\mathrm{ZnO}(15)$ calculated for primitive unit cell are $7.92 \mathrm{eV} / \AA^{2}$ (instead of 4 $\times 2.05=8.20$ due to numerical calculations performed in a large supercell) and $15.04 \mathrm{eV} / \AA^{2}$ (instead of $4 \times 3.88$ $=15.52$ ), respectively. These values are smaller than those corresponding to graphene (40.96 and $\left.72.52 \mathrm{eV} / \AA^{2}\right)$ and BN (34.02 and $\left.60.46 \mathrm{eV} / \AA^{2}\right)$ nanoribbons.

The calculated in-plane stiffness values $C$ for a- $\mathrm{ZnO}(9)$ and a- $\mathrm{ZnO}(15)$ are 3.75 and $3.71 \mathrm{eV} / \AA^{2}$, respectively. ${ }^{63}$ The calculated $C$ values of armchair graphene $(\mathrm{BN})$ nanoribbons for $n=9$ and $n=15$ are 18.45 (14.55) and 18.85 (14.66) eV/ $\AA^{2}$, respectively. Graphene and BN nanoribbons have significantly higher in-plane stiffness as compared to $\mathrm{a}-\mathrm{ZnO}$ nanoribbons. The difference mainly originates from the fact that a-ZnO honeycomb structure has larger unit cell than that of graphene and $\mathrm{BN}$ honeycomb structures. In view of the calculated $\kappa$ and $C$ values, both graphene and $\mathrm{BN}$ nanoribbons are stiffer than $\mathrm{ZnO}$ nanoribbons.

Figure 9 illustrates also the atomic structure of a- $\mathrm{ZnO}(9)$ and $\mathrm{a}-\mathrm{ZnO}(15)$ nanoribbons for three different values of applied strain. After the yielding point, the honeycomb structure is destroyed and the polygons being smaller and larger than hexagons form. In some cases, a net magnetic moment can develop in those polygons. The onset of plastic range and variation in atomic structures can depend on the periodic boundary conditions. These artifacts of periodic boundary conditions are eliminated to some extent by using larger supercells comprising several unit cells. Nonetheless, the plastic deformations shown in Figs. 9(a) and 9(b) have still large 


\section{Zigzag ZnO Ribbon}
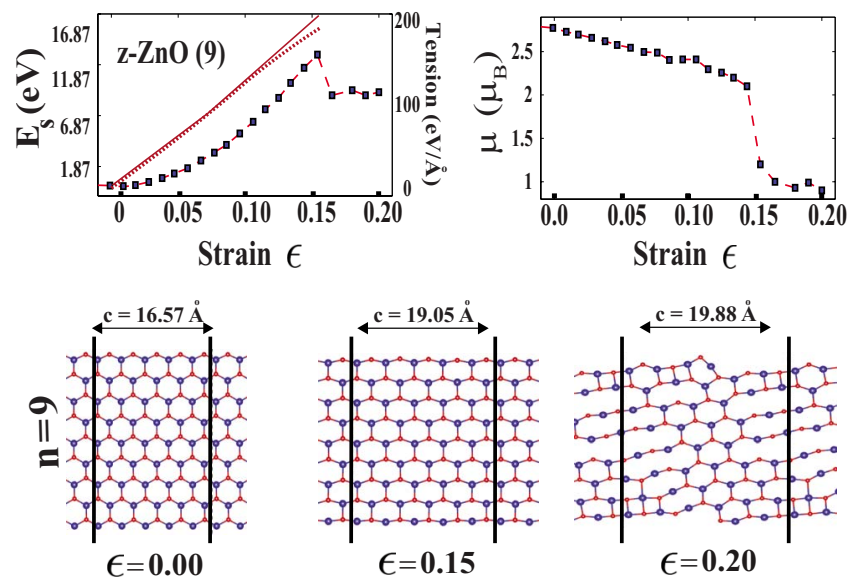

$\epsilon=0.15$

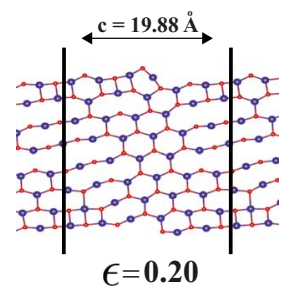

FIG. 10. (Color online) Variation in the strain energy $E_{s}$, tension, and magnetic moment $\mu$ per supercell of the bare $\mathrm{z}-\mathrm{ZnO}(n)$ $(n=9)$ with the applied uniaxial strain. Atomic structures of the bare $\mathrm{z}-\mathrm{ZnO}(9)$ for three different uniaxial strains. The supercells consist of five unit cells.

repeat periodicity. Even if the size and forms of polygons appear to be hysteric, their further investigation is of fundamental interest. The order-disorder structural transformation as well as dislocation generation appear to be absent in this study. The latter may require a treatment of deformation by taking into account very large $n$ and $c$. Moreover, as predicted for graphene nanoribbons, one expects that the onset of yielding in the presence of vacancy defects and also at high temperature can occur at small strain. ${ }^{57}$ Nevertheless, the plastic deformation in the present work is carried out under ideal conditions hence its stochastic nature is not taken into account. The energy gap of the nanoribbon undergoes a significant change under uniaxial tension. It decreases as $\epsilon$ increases and becomes very small at the yielding point. Beyond the yielding point the band gap increases again.

In Fig. 9(c) we plot the calculated values of force constant, $\kappa$ relative to the width $w$ of a- $\mathrm{ZnO}(n)$ taken as the distance between outermost atoms of both edges. As expected $\kappa$ is proportional to $w$ and $\kappa(w)$ can be considered as linear except small deviations from linearity due to edge effects. In fact, $\partial \kappa(w) / \partial w$ increases slightly with $w$. Whether a- $\mathrm{ZnO}(n)$ has even or odd $n$ also causes to a small deviation from single linear variation. As expected, the variation in the in-plane stiffness $C$ with $w$ is not significant. However, $C$ changes significantly between two subsequent values of $n$.

The zigzag $\mathrm{z}-\mathrm{ZnO}(9)$ nanoribbon displays a variation $E_{s}(\epsilon)$ similar to those of a-ZnO. The calculated value of $\kappa$ is $4.27 \mathrm{eV} / \AA^{2}$, which is larger than that calculated for a- $\mathrm{ZnO}(9)$ having relatively smaller width. Similarly, in-plane stiffness value of $\mathrm{z}-\mathrm{ZnO}(9)$ is slightly smaller than that of $\mathrm{a}-\mathrm{ZnO}(9)$ and $\mathrm{a}-\mathrm{ZnO}(15) . C$ is calculated as $3.24 \mathrm{eV} / \AA^{2}$. The zigzag $\mathrm{ZnO}(9)$ remains to be ferromagnetic metal in the elastic deformation range but their magnetic moment decreases with increasing $\epsilon$. However, beyond the yielding point it shows a sharp fall and diminish with increasing $\epsilon$ in the plastic range as seen in Fig. 10. In contrast to a- $\mathrm{ZnO}$, long polygons are aligned along the axis of the $\mathrm{z}-\mathrm{ZnO}(9)$ in Fig. 10.

\section{DISCUSSION AND CONCLUSIONS}

This work presents an extensive study on 2D monolayer and bilayer $\mathrm{ZnO}$, and $\mathrm{ZnO}$ nanoribbons together with their stability analysis. The monolayer of $\mathrm{ZnO}$ is an ionic and nonmagnetic, wide band-gap semiconductor with significant charge transfer from zinc atoms to nearest oxygen atoms. Since DFT normally underestimates the calculated band gaps, the calculated band gap of specific structures are corrected by $G W_{0}$ self-energy calculations. $\mathrm{ZnO}$ has $2 \mathrm{D}$ hexagonal lattice forming a planar honeycomb structure. Our predictions, which contribute to a better understanding of this material, are emphasized by way of conclusions: (i) we have shown that, 2D $\mathrm{ZnO}$ monolayer and bilayer, quasi-1D bare nanoribbons of $\mathrm{ZnO}$ and $\mathrm{OD}$ patch of $\mathrm{ZnO}$ correspond to local minima on the Born-Openheimer surface and thus are predicted to be stable. (ii) Ab initio molecular-dynamics calculations performed at high temperature corroborate our analysis obtained from the calculation of phonon frequencies. (iii) We performed calculations of phonon modes and density of states of phonon frequencies of $2 \mathrm{D}$ monolayer, quasi-1D armchair nanoribbon and a finite flake using forceconstant method. In particular, we revealed the acoustical twisting mode of armchair nanoribbon. (iv) Our study of $\mathrm{Zn}$, $\mathrm{O}, \mathrm{Zn}-\mathrm{O}$ vacancies, and $\mathrm{O}-\mathrm{Zn}$ antisite indicates that local magnetic moments can be generated only by $\mathrm{Zn}$ vacancies. (v) We provided an extensive analysis of the electronic structure of hydrogen terminated as well as bare armchair and zigzag nanoribbons. Armchair $\mathrm{ZnO}$ nanoribbons are found to be nonmagnetic semiconductors. The band gaps vary with their widths. The narrow nanoribbons have relatively larger band gap due to the quantum-confinement effect. Bare zigzag $\mathrm{ZnO}$ nanoribbons are ferromagnetic metals. The atoms near the oxygen-terminated edge of the ribbons acquire magnetic moments. The spin polarization at the Fermi level may attain high values for specific zigzag nanoribbons. However, once $\mathrm{O}$ - and $\mathrm{Zn}$-terminated edges are passivated with hydrogen atoms, the zigzag nanoribbon becomes nonmagnetic metal. These electronic and magnetic properties might be useful for spintronic applications. (vi) We found the minimum-energy stacking of the $\mathrm{ZnO}$ bilayer and provided energetics and energy band structure corresponding to this stacking. Accordingly, bilayer $\mathrm{ZnO}$ is also a nonmagnetic, wide band-gap semiconductor with slightly smaller band gap as compared to that of monolayer. (vii) We provided an analysis of mechanical properties. $\mathrm{ZnO}$ nanoribbons under uniaxial strain show harmonic and anharmonic elastic deformation ranges and a yielding point. After yielding, the strain energy exhibits a sharp fall and the nanoribbon deform plastically. In the elastic range, hexagons are uniformly deformed but honeycomblike atomic structure is maintained. After yielding point some of the hexagons are modified and reconstruct to different polygons which may be smaller or larger than hexagons. Variation in electronic and magnetic properties with deformation and formation of polygons in the plastic-deformation range are of fundamental interest. Calculation of force constants and in-plane stiffness indicate that the stiffness of $\mathrm{ZnO}$ nanoribbons is smaller than those of graphene and $\mathrm{BN}$ honeycomb structures. 
In summary, it is shown that single and bilayer $\mathrm{ZnO}$ and its nanoribbons in different orientations provide us for a variety of electronic and magnetic properties which may be interesting for further applications. Even if they have honeycomb structure common to monolayer graphene and $\mathrm{BN}$, their properties exhibit important differences.

\section{ACKNOWLEDGMENTS}

We acknowledge stimulating discussions with Ethem Akturk and Haldun Sevincli. Part of the computations have been provided by UYBHM at Istanbul Technical University through Grant No. 2-024-2007. This work is partially supported by TUBA, Academy of Science of Turkey.
${ }^{1}$ K. S. Novoselov, A. K. Geim, S. V. Morozov, D. Jiang, Y. Zhang, S. V. Dubonos, I. V. Grigorieva, and A. A. Firsov, Science 306, 666 (2004).

${ }^{2}$ Y. Zhang, Y.-W. Tan, H. L. Stormer, and P. Kim, Nature (London) 438, 201 (2005).

${ }^{3}$ C. Berger, Z. Song, X. Li, X. Wu, N. Brown, C. Naud, D. Mayou, T. Li, J. Hass, A. N. Marchenkov, E. H. Conrad, P. N. First, and W. A. de Heer, Science 312, 1191 (2006).

${ }^{4}$ K. S. Novoselov, D. Jiang, F. Schedin, T. Booth, V. V. Khotkevich, S. Morozov, and A. K. Geim, Proc. Natl. Acad. Sci. U.S.A. 102, 10451 (2005).

${ }^{5}$ Y. W. Son, M. L. Cohen, and S. G. Louie, Phys. Rev. Lett. 97, 216803 (2006).

${ }^{6}$ Y.-W. Son, M. L. Cohen, and S. G. Louie, Nature (London) 444, 347 (2006).

${ }^{7}$ M. Y. Han, B. Özyilmaz, Y. Zhang, and P. Kim, Phys. Rev. Lett. 98, 206805 (2007).

${ }^{8}$ H. Sevinçli, M. Topsakal, E. Durgun, and S. Ciraci, Phys. Rev. B 77, 195434 (2008).

${ }^{9}$ C.-H. Park and S. G. Louie, Nano Lett. 8, 2200 (2008).

${ }^{10}$ V. Barone and J. E. Peralta, Nano Lett. 8, 2210 (2008).

${ }^{11}$ M. Topsakal, E. Akturk, and S. Ciraci, Phys. Rev. B 79, 115442 (2009).

${ }^{12}$ S. Cahangirov, M. Topsakal, E. Akturk, H. Sahin, and S. Ciraci, Phys. Rev. Lett. 102, 236804 (2009).

${ }^{13}$ H. Şahin, S. Cahangirov, M. Topsakal, E. Bekaroglu, E. Akturk, R. T. Senger, and S. Ciraci, Phys. Rev. B 80, 155453 (2009).

${ }^{14}$ F. Claeyssens et al., J. Mater. Chem. 15, 139 (2005).

${ }^{15}$ A. J. Kulkarni, M. Zhou, K. Sarasamak, and S. Limpijumnong, Phys. Rev. Lett. 97, 105502 (2006).

${ }^{16}$ Y. Wang, X. Fan, and J. Sun, Mater. Lett. 63, 350 (2009).

${ }^{17}$ Y. Huang, J. He, Y. Zhang, Y. Dai, Y. Gu, S. Wang, and C. Zou, J. Mater. Sci. 41, 3057 (2006).

${ }^{18}$ J. Duan, X. Huang, and E. Wang, Mater. Lett. 60, 1918 (2006).

${ }^{19}$ G. S. Wu, T. Xie, X. Y. Yuan, Y. Li, L. Yang, Y. H. Xiao, and L. D. Zhang, Solid State Commun. 134, 485 (2005).

${ }^{20}$ C. Tusche, H. L. Meyerheim, and J. Kirschner, Phys. Rev. Lett. 99, 026102 (2007).

${ }^{21}$ D.-K. Hwang, M.-S. Oh, J. Lim, and S.-J. Park, J. Phys. D 40, R387 (2007).

${ }^{22}$ M. Law, L. E. Greene, J. C. Johnson, R. Saykally, and P. Yang, Nature Mater. 4, 455 (2005).

${ }^{23}$ Z. Q. Chen, M. Maekawa, A. Kawasuso, S. Sakai, and H. Naramoto, Physica B 376-377, 722 (2006)

${ }^{24}$ Q. Wang, Q. Sun, G. Chen, Y. Kawazoe, and P. Jena, Phys. Rev. B 77, 205411 (2008).

${ }^{25}$ P. E. Blöchl, Phys. Rev. B 50, 17953 (1994).

${ }^{26}$ J. P. Perdew, J. A. Chevary, S. H. Vosko, K. A. Jackson, M. R. Pederson, D. J. Singh, and C. Fiolhais, Phys. Rev. B 46, 6671
(1992).

${ }^{27}$ The capacity of spin-polarized DFT and its comparison with other well-known methods can be found in a review article: "Spin-Polarized DFT Calculations and Magnetism" by R. Zeller in Computational Nanoscience: Do It Yourself, NIC Series, edited by J. Grotendorst, S. Blugel, and D. Marx (John von Neumann Institute for Computing, Julich, 2006), Vol. 31, pp. 419445.

${ }^{28}$ G. Kresse and J. Hafner, Phys. Rev. B 47, 558 (1993).

${ }^{29}$ G. Kresse and J. Furthmuller, Phys. Rev. B 54, 11169 (1996).

${ }^{30}$ S. Baroni, A. Del Corso, S. Girancoli, and P. Giannozzi, http:/ www.pwscf.org/

${ }^{31}$ J. M. Soler, E. Artacho, J. D. Gale, A. Garcia, J. Junquera, P. Ordejon, and D. Sanchez-Portal, J. Phys.: Condens. Matter 14, 2745 (2002). Calculations are performed within GGA. A double- $\zeta$ plus polarization basis set was used. A charge-density cutoff of 400 Ry was used.

${ }^{32}$ M. Shishkin and G. Kresse, Phys. Rev. B 74, 035101 (2006).

${ }^{33}$ Ü. Özgür, Ya. I. Alivov, C. Liu, A. Teke, M. A. Reshchikov, S. Doğan, V. Avrutin, S.-J. Cho, and H. Morkoç, J. Appl. Phys. 98, 041301 (2005).

${ }^{34}$ M. Catti, Y. Noel, and R. Doves, J. Phys. Chem. Solids 64, 2183 (2003); see also J. E. Jaffe and A. C. Hess, Phys. Rev. B 48 , 7903 (1993).

${ }^{35}$ J. Hafner, J. Comput. Chem. 29, 2044 (2008).

${ }^{36}$ M. Oshikiri and F. Aryasetiawan, Phys. Rev. B 60, 10754 (1999).

${ }^{37}$ M. Usuda, N. Hamada, T. Kotani, and M. vanSchilfgaarde, Phys. Rev. B 66, 125101 (2002).

${ }^{38}$ P.-O. Löwdin, J. Chem. Phys. 18, 365 (1950).

${ }^{39} \mathrm{G}$. Henkelman, A. Arnaldsson, and H. Jonsson, Comput. Mater. Sci. 36, 354 (2006).

${ }^{40}$ Calculated effective charge of $\mathrm{Zn}$ atom indicating $\sim 1$ electron occupancy of the valance orbitals eliminates the inert gas configuration. Therefore, one considers the covalent radii, namely, $R_{\mathrm{Zn}}=1.31 \AA$ and $R_{\mathrm{O}}=0.66 \AA$.

${ }^{41}$ R. E. Peierls, Ann. Inst. Henri Poincare 5, 177 (1935).

${ }^{42}$ L. D. Landau, Phys. Z. Sowjetunion 11, 26 (1937).

${ }^{43}$ D. Alfè, Comput. Phys. Commun. 180, 2622 (2009).

${ }^{44}$ Z. C. Tu, arXiv:0901.1112, J. Comput. Theor. Nanosci. (to be published).

${ }^{45}$ P. Esquinazi, D. Spemann, R. Höhne, A. Setzer, K.-H. Han, and T. Butz, Phys. Rev. Lett. 91, 227201 (2003).

${ }^{46}$ A. Hashimoto, K. Suenaga, A. Gloter, K. Urita, and S. Iijima, Nature (London) 430, 870 (2004).

${ }^{47}$ O. V. Yazyev and L. Helm, Phys. Rev. B 75, 125408 (2007).

${ }^{48}$ M. A. H. Vozmediano, M. P. Lopez-Sancho, T. Stauber, and F. Guinea, Phys. Rev. B 72, 155121 (2005).

${ }^{49}$ L. Brey, H. A. Fertig, and S. Das Sarma, Phys. Rev. Lett. 99, 
116802 (2007).

${ }^{50}$ M. Topsakal, H. Sevinçli, and S. Ciraci, Appl. Phys. Lett. 92, 173118 (2008).

${ }^{51}$ E. H. Lieb, Phys. Rev. Lett. 62, 1201 (1989).

${ }^{52}$ R. Gillen, M. Mohr, J. Maultzsch, and C. Thomsen, Phys. Rev. B 80, 155418 (2009).

${ }^{53}$ The variation in $E_{G}$ with $n$ shows an family-dependent behavior for armchair nanoribbons of graphene, BN, and even Si (Ref. 13). For example, the band gap is small for $n=3 m-1$ ( $m$ being an integer) but from $n=3 m$ to $n=3 m+1$ it increases and passing through a maximum it becomes again small at the next minimum corresponding to $n=3 m-1$ for armchair graphene nanoribbons.

${ }^{54}$ A. R. Botello-Mendez, F. Lopez-Urias, M. Terrones, and H. Terrones, Nano Lett. 8, 1562 (2008).

${ }^{55}$ A. R. Botello-Méndez, M. T. Martínez-Martínez, F. López-Urías, M. Terrones, and H. Terrones, Chem. Phys. Lett. 448, 258 (2007).

${ }^{56}$ Z. H. Ni, T. Yu, Y. H. Lu, Y. Y. Wang, Y. P. Feng, and Z. X. Shen, ACS Nano 2, 2301 (2008); V. M. Pereira and A. H. Castro Neto, Phys. Rev. Lett. 103, 046801 (2009); F. Guinea, M. I. Kartsnelson, and K. M. Geim, Nat. Phys. (to be published).

${ }^{57}$ M. Topsakal and S. Ciraci, arXiv:0907.0501 (unpublished).
${ }^{58}$ C. Jin, F. Lin, K. Suenaga, and S. Iijima, Phys. Rev. Lett. 102, 195505 (2009).

${ }^{59}$ B. I. Yakobson, C. J. Brabec, and J. Bernholc, Phys. Rev. Lett. 76, 2511 (1996).

${ }^{60}$ K. N. Kudin, G. E. Scuseria, and B. I. Yakobson, Phys. Rev. B 64, 235406 (2001).

${ }^{61}$ G. Dereli and C. Ozdogan, Phys. Rev. B 67, 035416 (2003).

${ }^{62}$ H. Mehrez and S. Ciraci, Phys. Rev. B 56, 12632 (1997). Through sequential order-disorder transformations a neck develops while the nanowire elongates.

${ }^{63}$ Because of uncertainties in measuring the width of a bare armchair nanoribbon, the value of $C$ cannot be determined unambigiously. For example, by taking $w$ as the distance between $\mathrm{Zn}$ atoms at both edges, $w=12.186 \AA$; alternatively $w=13.393 \AA$ if the width is taken as the distance between $\mathrm{O}$ atoms at both edges of $\mathrm{a}-\mathrm{ZnO}(9)$. The corresponding in-plane stiffness values are 3.977 and $3.618 \mathrm{eV} / \AA^{2}$, respectively. One obtains $C$ $\cong 3.75 \mathrm{eV} / \AA^{2}$. In conclusions, while the value of $C$ can be unambiguously determined for $2 \mathrm{D}$ periodic $\mathrm{ZnO}$ honeycomb structure, the value of $C$ calculated for a nanoribbon depends on its width. As $w$ increases, $C$ decreases, and eventually as $w \rightarrow \infty C$ approaches the value calculated for $2 \mathrm{D}$ periodic $\mathrm{ZnO}$ honeycomb structure. 